\title{
FlavBit: a GAMBIT module for computing flavour observables and likelihoods
}

\author{
The GAMBIT Flavour Workgroup: Florian U. Bernlochner' ${ }^{1}$, Marcin Chrzaszcz ${ }^{2,3, a}$, Lars A. Dal ${ }^{4}$, Ben Farmer ${ }^{5,6}$, \\ Paul Jackson $^{7,8}$, Anders Kvellestad ${ }^{9}$, Farvah Mahmoudi ${ }^{10,11, b, e}$, Antje Putze ${ }^{12}$, Christopher Rogan ${ }^{13}$, \\ Pat Scott $^{14, c}{ }_{(1)}$, Nicola Serra ${ }^{2, d}$, Christoph Weniger ${ }^{15}$, Martin White ${ }^{7,8}$ \\ ${ }^{1}$ Physikalisches Institut der Rheinischen Friedrich-Wilhelms-Universität Bonn, 53115 Bonn, Germany \\ 2 Physik-Institut, Universität Zürich, Winterthurerstrasse 190, 8057 Zurich, Switzerland \\ ${ }^{3}$ H. Niewodniczański Institute of Nuclear Physics, Polish Academy of Sciences, 31-342 Kraków, Poland \\ ${ }^{4}$ Department of Physics, University of Oslo, 0316 Oslo, Norway \\ 5 Oskar Klein Centre for Cosmoparticle Physics, AlbaNova University Centre, 10691 Stockholm, Sweden \\ ${ }^{6}$ Department of Physics, Stockholm University, 10691 Stockholm, Sweden \\ ${ }^{7}$ Department of Physics, University of Adelaide, Adelaide, SA 5005, Australia \\ ${ }^{8}$ Australian Research Council Centre of Excellence for Particle Physics at the Tera-scale, Australia, http://www.coepp.org.au/ \\ ${ }^{9}$ NORDITA, Roslagstullsbacken 23, 10691 Stockholm, Sweden \\ ${ }^{10}$ Univ Lyon, Univ Lyon 1, ENS de Lyon, CNRS, Centre de Recherche Astrophysique de Lyon UMR5574, 69230 Saint-Genis-Laval, France \\ 11 Theoretical Physics Department, CERN, 1211 Geneva 23, Switzerland \\ 12 LAPTh, Université de Savoie, CNRS, 9 chemin de Bellevue, B.P.110, 74941 Annecy-le-Vieux, France \\ 13 Department of Physics, Harvard University, Cambridge, MA 02138, USA \\ 14 Department of Physics, Blackett Laboratory, Imperial College London, Prince Consort Road, London SW7 2AZ, UK \\ 15 GRAPPA, Institute of Physics, University of Amsterdam, Science Park 904, 1098 XH Amsterdam, The Netherlands
}

Received: 18 April 2017 / Accepted: 22 August 2017 / Published online: 21 November 2017

(C) The Author(s) 2017. This article is an open access publication

\begin{abstract}
Flavour physics observables are excellent probes of new physics up to very high energy scales. Here we present FlavBit, the dedicated flavour physics module of the globalfitting package GAMBIT. FlavBit includes custom implementations of various likelihood routines for a wide range of flavour observables, including detailed uncertainties and correlations associated with $\mathrm{LHCb}$ measurements of rare, leptonic and semileptonic decays of $B$ and $D$ mesons, kaons and pions. It provides a generalised interface to external theory codes such as Superlso, allowing users to calculate flavour observables in and beyond the Standard Model, and then test them in detail against all relevant experimental data. We describe FlavBit and its constituent physics in some detail, then give examples from supersymmetry and effective field theory illustrating how it can be used both as a standalone library for flavour physics, and within GAMBIT.
\end{abstract}

\footnotetext{
a e-mail:mchrzasz@cern.ch

b e-mail: nazila@cern.ch

c e-mail: p.scott@imperial.ac.uk

d e-mail: nicola.serra@cern.ch

e Also Institut Universitaire de France, 103 boulevard Saint-Michel, 75005 Paris, France
}

\section{Contents}

1 Introduction ............... . . 2

2 Theoretical framework . . . . . . . . . . 2

3 Computational framework .......... . 3

4 Observables ............... . . 3

4.1 Interfaces to external codes . . . . . . . . . 4

4.2 Tree-level leptonic and semi-leptonic decays . . 4

4.3 Electroweak penguin transitions . . . . . . . 7

4.4 Rare purely leptonic decays . . . . . . . . . . . 10

4.5 Other flavour observables . . . . . . . . . 10

5 Likelihoods . . . . . . . . . . . . . . 12

5.1 Tree-level leptonic and semi-leptonic likelihood 13

5.2 Electroweak penguin likelihood ... . . . . 14

5.3 Rare purely leptonic likelihood . . . . . . . . . 15

5.4 Rare radiative $B$ decay likelihood . . . . . . 15

$5.5 B$ meson mass asymmetry likelihood . . . . . 16

5.6 Other observables . . . . . . . . . . . 16

6 Examples .................. 16

6.1 Supersymmetric scan ........... 16

6.2 Wilson coefficient fit . . . . . . . . . . 17

6.3 FlavBit standalone example . . . . . . . . 17

7 Conclusions ............... . 19 
Appendix A: Glossary . . . . . . . . . . . . . . . . . . 19

References ............... 20

\section{Introduction}

Precise measurement of flavour observables is a powerful indirect probe of physics beyond the Standard Model (SM), as new heavy particles predicted by extensions of the SM can contribute to the amplitudes of observables as virtual particles. Flavour observables are therefore sensitive to much higher energy scales than direct searches for new particles. Moreover, rare decays, such as Flavour Changing Neutral Currents (FCNCs), are loop suppressed in the SM. As a consequence, the SM decay rates are small, and could be comparable in magnitude to contributions from new heavy states, allowing stringent constraints to be placed on the parameters of theories for new physics. It is therefore crucial to consider constraints from flavour physics when studying scenarios beyond the SM. The correlations between the different flavour observables, and the interplay between flavour measurements and direct searches at collider experiments, are key tools in the search for new physics, and its eventual understanding.

Public packages exist for carrying out SM and BSM flavour fits in terms of Wilson coefficients [1-3], but so far no general package exists for both computing Wilson coefficients and carrying out a global fit. In this article we present FlavBit, a flavour physics library designed in the context of the Global And Modular BSM Inference Tool (GAMBIT) framework [4], but also usable in standalone form. FlavBit allows users to predict flavour physics observables in various models, using external programs such as Superlso [5-7], and then calculate combined likelihoods for arbitrary combinations of the observables. FlavBit takes into account all theoretical and experimental correlations between the different observables. The resulting likelihoods can be incorporated into the GAMBIT global likelihood to scan the parameter spaces of various models for new physics [4,8-11], taking into account complementary constraints from direct production [12], dark matter searches [13], and SM and related precision measurements [14].

Recently, some measurements of flavour observables, mainly from LHCb [15-18] and $B$ factories [19-23], have shown tension with their predicted values in the SM. It is still unclear if these might be accommodated in the SM by larger-than-expected QCD effects, statistical fluctuations or some combination thereof. Nonetheless, these tensions certainly provide motivation for continued interest and effort in careful combination and cross-correlation of flavour observables with each other, and with searches for new physics in other sectors. We include these measurements in FlavBit.
This paper is organised as follows. In Sect. 2 we provide the general theoretical background of the scheme by which we compute flavour observables, before providing a brief synopsis in Sect. 3 of the broader global-fitting framework within which FlavBit sits. In Sect. 4 we discuss the predictions and measurements of individual observables included in FlavBit 1.0.0, and highlight aspects of new physics models to which the different measurements are sensitive. Section 5 gives details of the likelihood calculations that FlavBit performs. Section 6 gives some usage examples, both in standalone mode and with GAMBIT proper. Section 7 summarises our conclusions, and Appendix A gives a glossary of relevant GAMBIT terminology helpful for reading this paper.

The FlavBit source code is freely available from gambit.hepforge.org under the terms of the standard 3-clause BSD license. ${ }^{1}$

\section{Theoretical framework}

Our theoretical framework for studying rare decay observables is based on the effective Hamiltonian approach, which provides a simple formulation that can be easily extended to incorporate contributions from new physics. In this formulation, the low- and high-energy effects are separated using the Operator Product Expansion method. Cross-sections for transitions from initial states $i$ to final states $f$ are proportional to squared matrix elements $\left|\left\langle f\left|\mathcal{H}_{\text {eff }}\right| i\right\rangle\right|^{2}$, where the effective Hamiltonian $\mathcal{H}_{\text {eff }}$ for $b \rightarrow s$ transitions is given by

$\mathcal{H}_{\text {eff }}=-\frac{4 G_{F}}{\sqrt{2}} V_{t b} V_{t s}^{*} \sum_{i=1}^{10}\left(C_{i}(\mu) \mathcal{O}_{i}(\mu)+C_{i}^{\prime}(\mu) \mathcal{O}_{i}^{\prime}(\mu)\right)$.

Here $G_{\mathrm{F}}$ is the Fermi constant, $\mu$ is the energy scale at which calculations are to be performed, and $V_{t b}$ and $V_{t s}$ are the usual CKM matrix elements. The $C_{i}$ are Wilson coefficients, which incorporate the influence of small-scale physics due to heavy states that have been integrated out in the effective theory; their values can be calculated using perturbative methods. The $\mathcal{O}_{i}$ are local operators representing long-distance interactions. The most relevant operators for the FCNC rare $B$ decays are

$$
\begin{aligned}
& \mathcal{O}_{1}=\left(\bar{s} \gamma_{\mu} T^{a} P_{L} c\right)\left(\bar{c} \gamma^{\mu} T^{a} P_{L} b\right), \\
& \mathcal{O}_{2}=\left(\bar{s} \gamma_{\mu} P_{L} c\right)\left(\bar{c} \gamma^{\mu} P_{L} b\right),
\end{aligned}
$$

\footnotetext{
${ }^{1}$ http://opensource.org/licenses/BSD-3-Clause. Note that fjcore [24] and some outputs of FlexibleSUSY [25] (incorporating routines from SOFTSUSY [26]) are also shipped with GAMBIT 1.0. These code snippets are distributed under the GNU General Public License (GPL; http://opensource.org/licenses/GPL-3.0), with the special exception, granted to GAMBIT by the authors, that they do not require the rest of GAMBIT to inherit the GPL.
} 


$$
\begin{aligned}
\mathcal{O}_{3} & =\left(\bar{s} \gamma_{\mu} P_{L} b\right) \sum_{q}\left(\bar{q} \gamma^{\mu} q\right), \\
\mathcal{O}_{4} & =\left(\bar{s} \gamma_{\mu} T^{a} P_{L} b\right) \sum_{q}\left(\bar{q} \gamma^{\mu} T^{a} q\right), \\
\mathcal{O}_{5} & =\left(\bar{s} \gamma_{\mu_{1}} \gamma_{\mu_{2}} \gamma_{\mu_{3}} P_{L} b\right) \sum_{q}\left(\bar{q} \gamma^{\mu_{1}} \gamma^{\mu_{2}} \gamma^{\mu_{3}} q\right), \\
\mathcal{O}_{6} & =\left(\bar{s} \gamma_{\mu_{1}} \gamma_{\mu_{2}} \gamma_{\mu_{3}} T^{a} P_{L} b\right) \sum_{q}\left(\bar{q} \gamma^{\mu_{1}} \gamma^{\mu_{2}} \gamma^{\mu_{3}} T^{a} q\right), \\
\mathcal{O}_{7} & =\frac{e}{(4 \pi)^{2}} m_{b}\left(\bar{s} \sigma^{\mu \nu} P_{R} b\right) F_{\mu \nu}, \\
\mathcal{O}_{8} & =\frac{g}{(4 \pi)^{2}} m_{b}\left(\bar{s} \sigma^{\mu \nu} T^{a} P_{R} b\right) G_{\mu \nu}^{a}, \\
\mathcal{O}_{9} & =\frac{e^{2}}{(4 \pi)^{2}}\left(\bar{s} \gamma^{\mu} P_{L} b\right)\left(\bar{\ell} \gamma_{\mu} \ell\right), \\
\mathcal{O}_{10} & =\frac{e^{2}}{(4 \pi)^{2}}\left(\bar{s} \gamma^{\mu} P_{L} b\right)\left(\bar{\ell} \gamma_{\mu} \gamma_{5} \ell\right),
\end{aligned}
$$

\section{Computational framework}

The GAMBIT framework defines two sorts of functions that can be used to calculate physical observables or other quantities required for computing them:

module functions: functions written in $\mathrm{C}++$ and contained within a GAMBIT module.

backend functions: external library functions provided by a backend, such as Superlso or FeynHiggs.

For ease of reference, here we highlight and link specific GAMBIT terms to their entries in the glossary, found in Appendix A.

When writing GAMBIT module functions, the author assigns each a capability, which describes what the function can calculate. This may be an observable, e.g. a particular branching fraction for a given rare $B$ decay, or a likelihood, e.g. the combined likelihood defined using a set of rare decays. Module functions can be declared to have dependencies on the results of other module functions, which they indicate by specifying the capability of the module function that must be used to fill the dependency. Dependencies may be filled by any function within GAMBIT that has the requisite capability, whether or not it is part of the same GAMBIT module as the dependent function. Module functions may also have backend requirements, which are satisfied by functions from backend libraries. For example, in FlavBit 1.0.0, Superlso supplies many of the backend requirements of the module functions that calculate observables.

FlavBit notifies GAMBIT of its available module functions and their capabilities, dependencies and backend requirements. The user tells GAMBIT that they want to compute a given set of observables and likelihoods in a given scan, and the GAMBIT Core identifies the necessary module functions and runs its dependency resolution routines. These hook the module functions up to each other and run them in an order that ensures that all dependencies are computed before the functions that depend on them. Full details of this process can be found in the main GAMBIT paper [4].

In standalone mode, users can just call the module functions of FlavBit directly, providing any required dependencies and backend requirements manually.

\section{Observables}

In this section we discuss the observables included in FlavBit and their relevance for searches for new physics. 
The most important observables are the rare decays $B \rightarrow$ $X_{s} \gamma, B_{s}^{0} \rightarrow \mu^{+} \mu^{-}$and $B^{0} \rightarrow K^{* 0} \mu^{+} \mu^{-}$, as well as tree level decays such as $B^{ \pm} \rightarrow \tau v_{\tau}$ and $B \rightarrow D^{(*)} \ell v_{\ell}{ }^{2}$

Here we discuss the calculation of the different observables in four groups: tree-level leptonic and semi-leptonic decays (Sect. 4.2), electroweak penguin transitions (Sect. 4.3), rare purely leptonic decays (Sect. 4.4), and other flavour observables (Sect. 4.5). In these sections we outline the calculations required to predict each observable from theory; further details can be found in Ref. [6]. While for simplicity we present only the leading order expressions in this paper, in FlavBit itself we use the full calculations at the highest available accuracy.

The tree-level category includes $B$ and $D$ decays to leptons with an accompanying hadron and/or a neutrino in the final state. Observables in this category are the branching fractions for processes such as $B^{ \pm} \rightarrow \tau \nu_{\tau}, B \rightarrow D^{(*)} \tau \nu_{\tau}$ and $B \rightarrow D^{(*)} \ell \nu_{\ell}$. The electroweak penguin category includes the rare decays $B \rightarrow M \ell^{+} \ell^{-}$(with $M$ another meson lighter than the $B$ ), in particular the angular observables of the decay $B^{0} \rightarrow K^{* 0} \mu^{+} \mu^{-}$. The rare fully-leptonic category includes $B$ decays with only leptons in the final state, such as $B_{(s)}^{0} \rightarrow \mu^{+} \mu^{-}$. The fourth and final category includes $b \rightarrow s$ transitions in the radiative decays $B \rightarrow X_{s} \gamma$, the mass difference between the heavy $B_{H}$ and light $B_{L}$ eigenstates of the $B_{s}^{0}$ system $\left(\Delta M_{S}\right)$, and decays of kaons and pions, in particular the leptonic decay ratio $\mathcal{B}\left(K^{ \pm} \rightarrow \mu v_{\mu}\right) / \mathcal{B}\left(\pi^{ \pm} \rightarrow \mu v_{\mu}\right)$. Note that FlavBit does not incorporate the anomalous magnetic moment of the muon, as this is dealt with in PrecisionBit [14].

\subsection{Interfaces to external codes}

Theoretical predictions of observables in FlavBit are predominantly obtained through interfaces to external codes. Some predictions of flavour observables are available from FeynHiggs [27], for the SM and minimal supersymmetric SM (MSSM). ${ }^{3}$ In FlavBit 1.0.0, most observable calculations refer to Superlso 3.6 [5-7].

The interface to Superlso operates via the function SI_fill (see Table 1), which provides the SuperIso_modelinfo. This function fills a Superlso

\footnotetext{
$\overline{{ }^{2} \text { Here } D^{(*)}, B^{ \pm}}$and $\ell$ are shorthand notations. The first indicates that we are referring to both $B \rightarrow D \ell v_{\ell}$ and $B \rightarrow D^{*} \ell \nu_{\ell}$, but as distinct processes. The same is true of the second notation, which indicates that we are referring to both the original process and its $\mathrm{CP}$ conjugate, distinctly. In contrast, when referring to specific rates, $\ell$ is typically used to indicate that the final state does not distinguish between $\ell=e$ and $\ell=\mu$. Some groups use this notation to refer to a sum over all final states involving electrons and muons, others use it to refer to the average. The PDG uses the former notation, which we follow in this paper except where explicitly noted otherwise.

3 The GAMBIT interface to FeynHiggs is described in detail in Sec 3.1.3 of Ref. [14].
}

parameters structure, which is passed back to various other Superlso functions to compute observables. Observables that are calculated directly from the input model parameters (Table 1) are distinguished from those that involve the calculation of intermediate Wilson coefficients (Tables 2, 3). In FlavBit 1.0.0, observables are implemented for MSSM models ('MSSM63atQ' and descendants; see [4]), and for a flavour EFT model ('WC') where the Wilson coefficients are specified directly as model parameters, and scanned over.

The design of FlavBit and its interface to Superlso make extending FlavBit to other models quite straightforward, either by computing Wilson coefficients 'upstream' from fundamental parameters, or by constructing the SuperIso_modelinfo to fit the model under investigation. SI_fill deals with the majority of the model-dependence in each calculation, importing different masses and couplings from SpecBit depending on the model being scanned, and using them to set various flags and member variables of the SuperIso_modelinfo.

SI_fill has a single option configurable from the master YAML file of a given scan: a boolean flag take_b_pole_mass_from_spectrum. This option allows the user to choose between Superlso's internal calculation of the $b$ quark pole mass (based on the $\overline{M S}$ mass imported from GAMBIT), or GAMBIT's own $b$ pole mass calculation provided by SpecBit [14]. Depending on the spectrum generator chosen in SpecBit, the standard 2-loop conversion from $\overline{M S}$ to pole mass included in Superlso may be a more accurate choice for precision $B$ physics than other calculations, even if the other calculation includes higher-order corrections. This is because the $b$ pole is sufficiently close to the QCD scale that problems with the perturbative expansion required to compute it start to show already at 3 loops [28], such that the formal error on the $b$ pole mass associated with truncating the asymptotic series may already be larger when truncating at 3 rather than 2 loops. This means that although 3-loop QCD RGEs remain preferable, 2-loop self energies give a more precise value for the $b$ pole, and should be preferred for $B$ physics calculations. In FlavBit 1.0.0, take_b_pole_mass_from_spectrum therefore defaults to false. 4

\subsection{Tree-level leptonic and semi-leptonic decays}

Decays of $B$ mesons with leptons and neutrinos in the final state proceed via tree-level charged currents. They have been intensively studied at $B$ factories (Babar, Belle and CLEO) for the determination of the elements $V_{c b}$ and $V_{u b}$ of the CKM matrix.

\footnotetext{
${ }^{4}$ Note that Superlso only actually uses the $b$ pole mass for computing the $1 \mathrm{~S}$ mass, which is better-behaved than the pole mass and preferable for observable calculations.
} 
Table 1 Observable capabilities of FlavBit that do not involve Wilson coefficients. Details of the fh_FlavourObs structure can be found in Table 5

\begin{tabular}{|c|c|c|c|}
\hline Capability & Function (return type): brief description & Dependencies (model) & Backend requirements \\
\hline SuperIso_modelinfo & $\begin{array}{l}\text { SI_fill (parameters): } \\
\text { Fills the Superlso structure. Key routine of the } \\
\text { Superlso interface }\end{array}$ & $\begin{array}{l}\text { MSSM_spectrum (MSSM63atQ) } \\
\text { SM_spectrum (WC) } \\
\text { W_plus_decay_rates } \\
\text { Z_decay_rates }\end{array}$ & $\begin{array}{l}\text { Init_param } \\
\text { slha_adjust } \\
\text { mb_1S }\end{array}$ \\
\hline Dstaunu & $\begin{array}{l}\text { SI_Dstaunu (double): } \\
\text { Computes the branching fraction of } D_{s}^{ \pm} \rightarrow \tau v_{\tau}\end{array}$ & SuperIso_modelinfo & Dstaunu \\
\hline Dsmunu & $\begin{array}{l}\text { SI_Dsmunu (double): } \\
\text { Computes the branching fraction of } D_{s}^{ \pm} \rightarrow \mu v_{\mu}\end{array}$ & SuperIso_modelinfo & Dsmunu \\
\hline Dmunu & $\begin{array}{l}\text { SI_Dmunu (double): } \\
\text { Computes the branching fraction of } D^{ \pm} \rightarrow \mu v_{\mu}\end{array}$ & SuperIso_modelinfo & Dmunu \\
\hline Btaunu & $\begin{array}{l}\text { SI_Btaunu (double): } \\
\text { Computes the branching fraction of } B^{ \pm} \rightarrow \tau v_{\tau}\end{array}$ & SuperIso_modelinfo & Btaunu \\
\hline BDtaunu & $\begin{array}{l}\text { SI_BDtaunu (double): } \\
\text { Computes the branching fraction of } B \rightarrow D \tau \nu_{\tau}\end{array}$ & SuperIso_modelinfo & BRBDlnu \\
\hline BDmunu & $\begin{array}{l}\text { SI_BDmunu (double): } \\
\text { Computes the branching fraction of } B \rightarrow D \mu v_{\mu}\end{array}$ & SuperIso_modelinfo & BRBDlnu \\
\hline BDstartaunu & $\begin{array}{l}\text { SI_BDstartaunu (double): } \\
\text { Computes the branching fraction of } B \rightarrow D^{*} \tau \nu_{\tau}\end{array}$ & SuperIso_modelinfo & BRBDstarlnu \\
\hline BDstarmunu & $\begin{array}{l}\text { SI_BDstarmunu (double): } \\
\text { Computes the branching fraction of } B \rightarrow D^{*} \mu v_{\mu}\end{array}$ & SuperIso_modelinfo & BRBDstarlnu \\
\hline $\mathrm{RD}$ & $\begin{array}{l}\text { SI_RD (double): } \\
\text { Computes the ratio } \mathcal{B}\left(B \rightarrow D \tau v_{\tau}\right) / \mathcal{B}\left(B \rightarrow D l v_{l}\right) \text {, } \\
\text { where } \ell=\mu \text { or } e \text { and the result is the same for each }\end{array}$ & SuperIso_modelinfo & BDtaunu_BDenu \\
\hline RDstar & $\begin{array}{l}\text { SI_RDstar }(\text { double }) \text { : } \\
\text { Computes the ratio } \\
\mathcal{B}\left(B \rightarrow D^{*} \tau \nu_{\tau}\right) / \mathcal{B}\left(B \rightarrow D^{*} l \nu_{l}\right) \text {, where } \ell=\mu \text { or } \\
e \text { and the result is the same for each }\end{array}$ & SuperIso_modelinfo & $\begin{array}{l}\text { BDstartaunu_ } \\
\text { BDstarenu }\end{array}$ \\
\hline Rmu & $\begin{array}{l}\text { SI_Rmu (double): } \\
\text { Computes the ratio } \mathcal{B}\left(K^{ \pm} \rightarrow \mu v_{\mu}\right) / \mathcal{B}\left(\pi^{ \pm} \rightarrow \mu v_{\mu}\right)\end{array}$ & SuperIso_modelinfo & Kmunu_pimunu \\
\hline Rmu23 & $\begin{array}{l}\text { SI_Rmu23 (double): } \\
\text { Computes the observable } R_{\mu 23} \text { (Eq. } 32 \text { ). }\end{array}$ & SuperIso_modelinfo & Rmu23 \\
\hline FH_FlavourObs & $\begin{array}{l}\text { FH_FlavourObs ( } f \text { h_FlavourObs): } \\
\text { Computes the FeynHiggs flavour observables }\end{array}$ & & FHFlavour \\
\hline deltaMs & $\begin{array}{l}\text { FH_DeltaMs (double): } \\
\text { Extracts the FeynHiggs MSSM prediction for the } \\
\quad B_{s}-\bar{B}_{S} \text { mass difference } \Delta M_{S}\left(\text { in }^{-1}\right)\end{array}$ & FH_FlavourObs & \\
\hline
\end{tabular}

The rate of the semi-leptonic decay $B \rightarrow M \ell v_{\ell}$ in the SM is

$\frac{\mathrm{d} \Gamma}{\mathrm{d} q^{2}}=\frac{G_{F}^{2}\left|V_{q b}^{2}\right|}{192 \pi^{3} m_{B}^{3}} \mathcal{K}\left(m_{B}^{2}, m_{M}^{2}, q^{2}\right) \mathcal{F}^{(2)}\left(q^{2}\right)$,

where $q^{\mu}=p_{B}^{\mu}-p_{M}^{\mu}$ is the momentum transfer, $V_{q b}$ is the CKM element corresponding to the flavour of $M, \mathcal{K}$ is a phase-space factor and $\mathcal{F}^{(2)}\left(q^{2}\right)$ is a combination of form factors [29].
These decays are sensitive to charged-current contributions from new particles. For example, the charged Higgs in the two Higgs doublet model (2HDM) (see e.g. Refs. [3033]), right-handed currents via the contribution of the charged mediator $W_{R}[32,34]$, new left-handed heavy bosons $W^{\prime}$ [35, 36] and leptoquarks (see e.g. Refs. [37,38]) can also modify the value of this observable.

The decays $B^{ \pm} \rightarrow \ell v_{\ell}$ also proceed via tree-level charged currents. The branching fraction is 


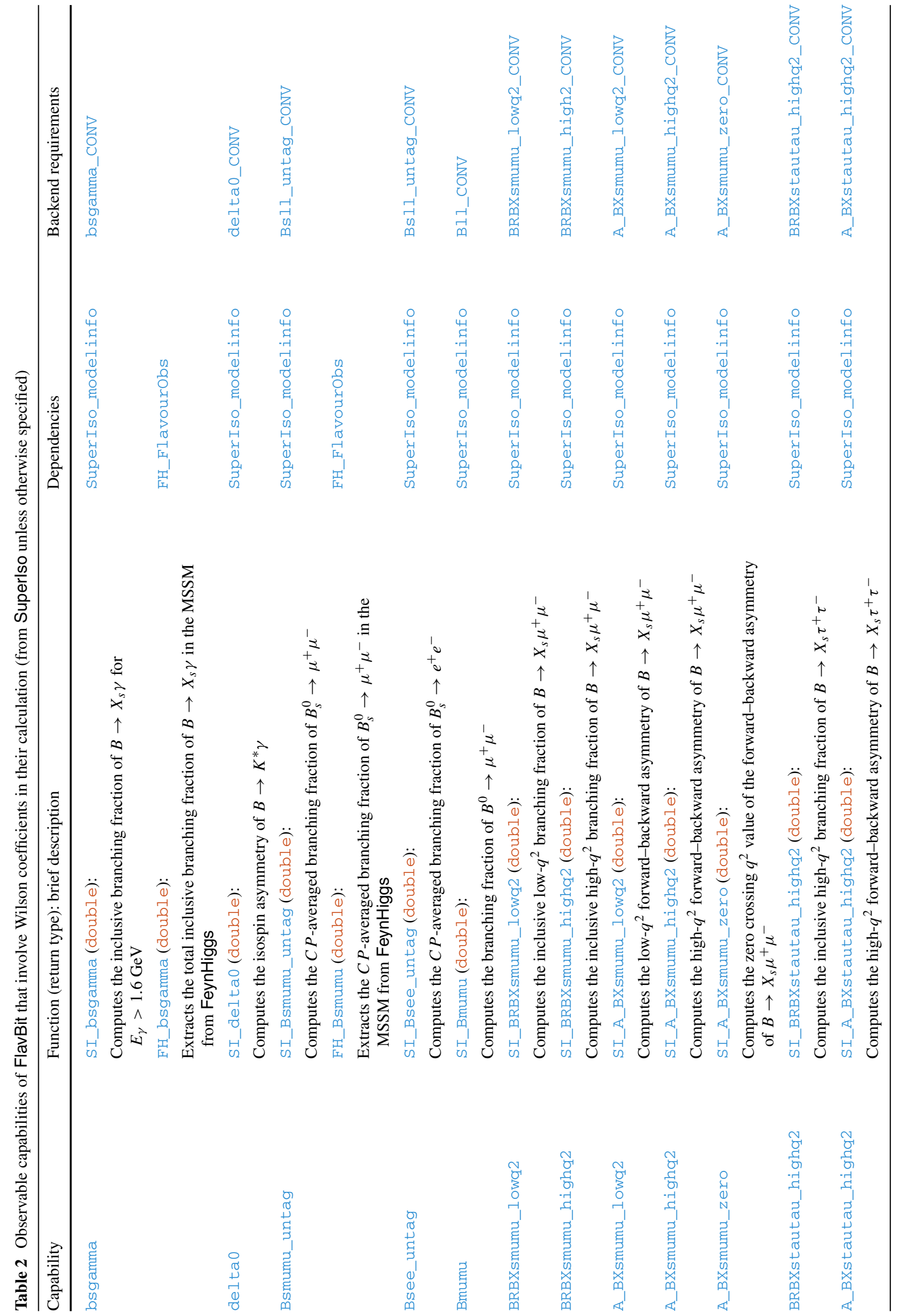


Table 3 Observable capabilities of FlavBit related to the decay $B^{0} \rightarrow$ $K^{* 0} \mu^{+} \mu^{-}$. The indices $l$ and $m$ refer to the edges of the energy bin used in the particular function. The functions and capabilities are named such that $l, m=11,25$ indicates an energy range of $1.1-2.5 \mathrm{GeV}^{2}$, and so on.
Possible pairs of $l$ and $m$ are $(11,25),(25,40),(40,60),(60,80),(15,17)$ and $(17,19)$; the last two refer to momentum transfer ranges of 15-17-19 $\mathrm{GeV}^{2}$

\begin{tabular}{|c|c|c|c|}
\hline Capability & Function (return type): brief description & Dependencies & Backend requirements \\
\hline BKstarmumu_l_m & $\begin{array}{l}\text { SI_BK,starmumu_l_m } \\
\text { (Flav_KstarMuMu_obs): } \\
\text { Computes all observables associated with } \\
B^{0} \rightarrow K^{* 0} \mu^{+} \mu^{-} \text {in a } q^{2} \text { bin specified by } l \text { and } m \text {. } \\
\text { See caption for details }\end{array}$ & SuperIso_modelinfo & SI_BKstarmumu_CONV \\
\hline AI_BKstarmumu & $\begin{array}{l}\text { SI_AI_BKstarmumu (double): } \\
\text { Computes the low- } q^{2} \text { isospin asymmetry of } \\
\quad B \rightarrow K^{*} \mu^{+} \mu^{-}\left(\text {in } \mathrm{GeV}^{2} \text { ) }\right.\end{array}$ & SuperIso_modelinfo & AI_BKstarmumu_CONV \\
\hline AI_BKstarmumu_zero & $\begin{array}{l}\text { SI_AI_BKstarmumu_zero (double): } \\
\text { Computes the zero-crossing } q^{2} \text { value of the isospin } \\
\text { asymmetry of } B \rightarrow K^{*} \mu^{+} \mu^{-}\end{array}$ & SuperIso_modelinfo & $\begin{array}{l}\text { AI_BKstarmumu_ } \\
\text { zero_CONV }\end{array}$ \\
\hline
\end{tabular}

$\mathcal{B}\left(B^{+} \rightarrow \ell^{+} v_{\ell}\right)=\frac{G_{F}^{2} m_{B} m_{\ell}^{2}}{8 \pi}\left(1-\frac{m_{\ell}^{2}}{m_{B}^{2}}\right)^{2} f_{B}^{2}\left|V_{u b}\right|^{2} \tau_{B}$,

where $f_{B}$ is the meson decay constant and $\tau_{B}$ is the lifetime of the $B^{+}$. This decay is sensitive to the CKM element $V_{u b}$. The charged Higgs sector of the 2HDM can again provide substantial contributions, as can new charged gauge bosons like the $W^{\prime}$ and $W_{R}$ of the left-right symmetric model [39]. Compared to the case where $\ell=\tau$, the decays with $\ell=e$ and $\ell=\mu$ have much smaller branching fractions, as they are helicity-suppressed. For this reason, at present only upper limits are available for the decays to light leptons. Although we provide routines to predict the values of all three in FlavBit, we only incorporate the tauonic version into the resulting likelihood.

Similarly, the decays $D_{(s)}^{ \pm} \rightarrow \ell v_{\ell}$ are mediated by the $W$ boson in the SM. The branching fractions can be obtained from Eq. 6 after the replacement $B \rightarrow D_{(s)}$ and swapping in the relevant CKM element. These decays have been traditionally used to measure the $D_{(s)}$ meson decay constant. However, the charged Higgs boson in the 2HDM would also mediate these decays, so they can provide complementary constraints to the analogous $B$ meson decay [40].

As shown in Table 1, FlavBit provides functions capable of computing branching fractions for $D_{s}^{ \pm} \rightarrow \tau v_{\tau}$ (Dstaunu), $D_{s}^{ \pm} \rightarrow \mu v_{\mu}$ (Dsmunu), $D^{ \pm} \rightarrow \mu v_{\mu}$ (Dmunu), $B^{ \pm} \rightarrow \tau v_{\tau}$ (Btaunu), $B \rightarrow D \tau v_{\tau}$ (BDtaunu), $B \rightarrow D \mu v_{\mu}$ (BDmunu), $B \rightarrow D^{*} \tau v_{\tau}$ (BDstartaunu) and $B \rightarrow D^{*} \mu v_{\mu}$ (BDstarmunu). It can also compute $R_{D^{(*)}} \equiv \mathcal{B}(B \rightarrow$ $\left.D^{(*)} \tau v_{\tau}\right) / \mathcal{B}\left(B \rightarrow D^{(*)} l v_{l}\right)$, designated by capabilities RD and RDstar. Here $\ell$ in $R_{D^{(*)}}$ refers to either $\mu$ or $e$, not their sum (the branching fractions $B \rightarrow D^{(*)} l v_{l}$ are identical for $e$ and $\mu$, as both are effectively massless in the $B$ system).

\subsection{Electroweak penguin transitions}

Rare semi-leptonic decays of $B$ mesons proceed via flavourchanging neutral currents (FCNCs) in electroweak penguin diagrams, and set stringent constraints on possible contributions from new physics. FlavBit includes predictions of various FCNC $b \rightarrow s$ transitions. These decays are all proportional to the elements $V_{t b}$ and $V_{t s}$ of the CKM matrix.

Rare decays of the type $B \rightarrow M \ell^{+} \ell^{-}$, with one meson $M$ in the final state, are sensitive to the Wilson coefficients $C_{9,10}^{(\prime)}$. In addition, when $M$ is a vector, such as the $K^{*}(892)$, these decays are also sensitive to the Wilson coefficients $C_{7}^{(\prime)}$.

The four-quark operators $\left(\mathcal{O}_{1 \ldots 6}\right)$ in the effective Hamiltonian also contribute to the penguin diagrams, resulting in expressions with the same structure as $\mathcal{O}_{7}$ and $\mathcal{O}_{9}$. They can therefore be reabsorbed and used to define effective Wilson coefficients $C_{7}^{\text {eff }}$ and $C_{9}^{\text {eff }}$ [41],

$C_{7}^{\mathrm{eff}}=C_{7}-\frac{1}{3} C_{3}-\frac{4}{9} C_{4}-\frac{20}{3} C_{5}-\frac{80}{9} C_{6}$,

$C_{9}^{\mathrm{eff}}=C_{9}+Y\left(q^{2}\right)$,

where $Y$ contains the short distance contributions from the four-quark operators $[42,43]$.

The most accessible of the $B \rightarrow M \ell^{+} \ell^{-}$decays at $\mathrm{LHCb}$ are those including final-state muons. The differential decay rate for $B \rightarrow M \mu^{+} \mu^{-}$, where $M$ is a pseudoscalar, is given at leading order by [44]:

$$
\begin{aligned}
\frac{\mathrm{d} \Gamma}{\mathrm{d} q^{2}}= & \frac{G_{F}^{2} \alpha^{2}\left|V_{t b} V_{t s}^{*}\right|^{2} m_{B}^{3}}{(2 \pi)^{10}} u\left(q^{2}\right)\left\{v\left(q^{2}\right)\left|C_{10} f_{+}\left(q^{2}\right)\right|^{2}\right. \\
& +4 \frac{m_{\mu}^{2}\left(m_{B}^{2}-m_{M}^{2}\right)^{2}}{q^{2} m_{B}^{4}}\left|C_{10} f_{0}\left(q^{2}\right)\right|^{2} \\
& \left.+\left|C_{9}^{\mathrm{eff}} f_{+}\left(q^{2}\right)+2 \frac{m_{b}+m_{s}}{m_{B}+m_{M}} C_{7}^{\mathrm{eff}} f_{T}\left(q^{2}\right)\right|^{2}\right\}
\end{aligned}
$$


where $u\left(q^{2}\right)$ and $v\left(q^{2}\right)$ are kinematic factors, and $f_{0}, f_{+}$and $f_{T}$ are $q^{2}$-dependent form factors.

If $M$ is a vector particle, the $B \rightarrow M \ell^{+} \ell^{-}$decays are completely described by the dilepton invariant mass squared $q^{2}$ and three angles $\left(\theta_{l}, \theta_{K}\right.$ and $\phi$; see Refs. [45,46] for definitions). Measurements of angular observables of the decays $B^{0} \rightarrow K^{*}(892) \mu^{+} \mu^{-}$and $B_{s}^{0} \rightarrow \phi \mu^{+} \mu^{-}$provide a better sensitivity to new physics than measurements of branching fractions. As a function of $q^{2}$ and the three angles, the differential decay rate for $B^{0} \rightarrow K^{* 0} \mu^{+} \mu^{-}$is

$$
\begin{aligned}
& \frac{1}{\Gamma} \frac{\mathrm{d}^{3}(\Gamma+\bar{\Gamma})}{\mathrm{d} \cos \theta_{\ell} \mathrm{d} \cos \theta_{K} \mathrm{~d} \phi}=\frac{9}{32 \pi}\left[\frac{3}{4}\left(1-F_{\mathrm{L}}\right) \sin ^{2} \theta_{K}\right. \\
& \quad+F_{\mathrm{L}} \cos ^{2} \theta_{K}+\frac{1}{4}\left(1-F_{\mathrm{L}}\right) \sin ^{2} \theta_{K} \cos 2 \theta_{\ell} \\
& \quad-F_{\mathrm{L}} \cos ^{2} \theta_{K} \cos 2 \theta_{\ell}+S_{3} \sin ^{2} \theta_{K} \sin ^{2} \theta_{\ell} \cos 2 \phi \\
& \quad+S_{4} \sin 2 \theta_{K} \sin 2 \theta_{\ell} \cos \phi+S_{5} \sin 2 \theta_{K} \sin \theta_{\ell} \cos \phi \\
& \quad+\frac{4}{3} A_{\mathrm{FB}} \sin ^{2} \theta_{K} \cos \theta_{\ell}+S_{7} \sin 2 \theta_{K} \sin \theta_{\ell} \sin \phi \\
& \left.\quad+S_{8} \sin 2 \theta_{K} \sin 2 \theta_{\ell} \sin \phi+S_{9} \sin ^{2} \theta_{K} \sin ^{2} \theta_{\ell} \sin 2 \phi\right],
\end{aligned}
$$

where $\bar{\Gamma}$ is the decay rate of the $\mathrm{CP}$ conjugate mode. The angular observable $F_{\mathrm{L}}$ is the longitudinal polarisation fraction of the $K^{*}$. The other observables are $S_{i}$, and the forwardbackward asymmetry $A_{\mathrm{FB}}$. The most sensitive experimental analyses assume that there are no scalar contributions (which are constrained by the branching fraction of $B_{s}^{0} \rightarrow \mu^{+} \mu^{-}$), and no tensor contributions. ${ }^{5}$ This assumption makes it possible to eliminate the observables $S_{1}^{c}, S_{1}^{s}, S_{2}^{c}$ and $S_{2}^{s}$ in favour of a single observable $F_{\mathrm{L}}$. The physical observables are sesquilinear combinations of the transversity amplitudes [49],

$$
\begin{aligned}
F_{\mathrm{L}} & =1-F_{T}=\frac{A_{0}^{2}}{A_{\|}^{2}+A_{\perp}^{2}+A_{0}^{2}}, \\
S_{3} & =\frac{1}{2} \frac{A_{\perp}^{L 2}-A_{\|}^{L 2}}{A_{\|}^{2}+A_{\perp}^{2}+A_{0}^{2}}+L \rightarrow R, \\
S_{4} & =\frac{1}{\sqrt{2}} \frac{\mathcal{R} e\left(A_{0}^{L *} A_{\|}^{L}\right)}{A_{\|}^{2}+A_{\perp}^{2}+A_{0}^{2}}+L \rightarrow R, \\
S_{5} & =\sqrt{2} \frac{\mathcal{R} e\left(A_{0}^{L *} A_{\perp}^{L}\right)}{A_{\|}^{2}+A_{\perp}^{2}+A_{0}^{2}}-L \rightarrow R, \\
A_{\mathrm{FB}} & =\frac{8}{3} \frac{\mathcal{R} e\left(A_{\perp}^{L *} A_{\|}^{L}\right)}{A_{\|}^{2}+A_{\perp}^{2}+A_{0}^{2}}-L \rightarrow R,
\end{aligned}
$$

5 Although Ref. [47] includes measurements free from these assumptions, using the Method of Moments [48], the resulting precision is about $15 \%$ less than in the likelihood fit.

$$
\begin{aligned}
& S_{7}=\sqrt{2} \frac{\mathcal{I} m\left(A_{0}^{L *} A_{\|}^{L}\right)}{A_{\|}^{2}+A_{\perp}^{2}+A_{0}^{2}}+L \rightarrow R, \\
& S_{8}=\frac{1}{\sqrt{2}} \frac{\mathcal{I} m\left(A_{0}^{L *} A_{\perp}^{L}\right)}{A_{\|}^{2}+A_{\perp}^{2}+A_{0}^{2}}+L \rightarrow R, \\
& S_{9}=\frac{\mathcal{I} m\left(A_{\perp}^{L *} A_{\|}^{L}\right)}{A_{\|}^{2}+A_{\perp}^{2}+A_{0}^{2}}-L \rightarrow R .
\end{aligned}
$$

The indices $\perp$, $\|$ and 0 refer to the $K^{*}(892)$ transversity amplitudes, while $L \rightarrow R$ refers to the chirality-flipped version of the previous term in each expression.

The amplitudes $A_{\perp, \|, 0}$ depend on form factors and Wilson coefficients, and can be written at leading order in QCD in the form:

$$
\begin{aligned}
A_{\perp}^{L, R} \propto & \left\{\left(C_{9}^{\mathrm{eff}}+C_{9}^{\mathrm{eff} \prime}\right) \mp\left(C_{10}+C_{10}^{\prime}\right) \frac{V\left(q^{2}\right)}{m_{B}+m_{K^{*}}}\right. \\
& \left.+\frac{2 m_{b}}{q^{2}}\left(C_{7}^{\mathrm{eff}}+C_{7}^{\mathrm{eff} \prime}\right) T_{1}\left(q^{2}\right)\right\}, \\
A_{\|}^{L, R} \propto & \left\{\left(C_{9}^{\mathrm{eff}}-C_{9}^{\mathrm{eff} \prime}\right) \mp\left(C_{10}-C_{10}^{\prime}\right) \frac{A_{1}\left(q^{2}\right)}{m_{B}+m_{K^{*}}}\right. \\
& \left.+\frac{2 m_{b}}{q^{2}}\left(C_{7}^{\mathrm{eff}}-C_{7}^{\mathrm{eff} \prime}\right) T_{2}\left(q^{2}\right)\right\}, \\
A_{0}^{L, R} \propto & \left\{\left[\left(C_{9}^{\mathrm{eff}}-C_{9}^{\mathrm{eff} \prime}\right) \mp\left(C_{10}-C_{10}^{\prime}\right)\right]\right. \\
& \times\left[( m _ { B } ^ { 2 } - m _ { K ^ { * } } ^ { 2 } - q ^ { 2 } ) \left(m_{B}+m_{K^{*}} A_{1}\left(q^{2}\right)\right.\right. \\
& \left.\left.-\lambda \frac{A_{2}\left(q^{2}\right)}{m_{B}+m_{K^{*}}}\right)\right]+2 m_{b}\left(C_{7}^{\mathrm{eff}}+C_{7}^{\mathrm{eff} \prime}\right) \\
\times & {\left[\left(m_{B}^{2}+3 m_{K^{*}}^{2}-q^{2}\right) T_{2}\left(q^{2}\right)\right.} \\
& \left.\left.-\frac{\lambda}{m_{B}^{2}-m_{K^{*}}^{2}} T_{3}\left(q^{2}\right)\right]\right\} .
\end{aligned}
$$

In the limit of large recoil (low $q^{2}$ ), the seven form factors $A_{1,2}, T_{1,2,3}$ and $V$ can be replaced by only two form factors $\xi_{\perp}$ and $\xi_{\|}$. This makes it possible to write a set of six observables that are independent of form factors in this approximation (see Ref. [50]). These are denominated ${ }^{6} P_{i}^{(\prime)}$, with $i \in[1,6]$. Some of these observables were independently proposed by other authors with a different name, e.g. $P_{1}=A_{T}^{(2)}[51], P_{2}=2 \times A_{T}^{R e}[52]$.

The observables $P_{i}$ can be written as ratios of the observables $F_{\mathrm{L}}$ and $S_{i}$, therefore if the full form factors $A_{1,2}, T_{1,2,3}$, $V$ [53] and their correlations are used it is equivalent to using the full set of $P_{i}$ observables. One of the most interesting measurements in these decays is the observable $P_{5}^{\prime}$, which shows a deviation with respect to the SM prediction

\footnotetext{
${ }^{6}$ Note that for historical reasons the observables $P_{4,5,6}^{\prime}$ carry a ${ }^{\prime}$.
} 
Table 4 Observables contained in the Flav_KstarMuMu_obs structure

\begin{tabular}{ll}
\hline Name (type) & Description \\
\hline BR (double) & Branching fraction \\
AFB (double) & Forward-backward asymmetry \\
FL (double) & Longitudinal fraction \\
S3 (double) & $S_{3}$ \\
S4 (double) & $S_{4}$ \\
S5 (double) & $S_{5}$ \\
S7 (double) & $S_{7}$ \\
S8 (double) & $S_{8}$ \\
S9 (double) & $S_{9}$ \\
q2_min $($ double) & $q^{2}$ bin lower edge \\
q2_max (double) & $q^{2}$ bin upper edge \\
\hline
\end{tabular}

of about $4 \sigma$ in the region $4<q^{2} / \mathrm{GeV}^{2}<8[18,23,47]$. The most accredited explanation for this deviation is a reduced $C_{9}^{\text {eff }}\left(q^{2}\right)$ Wilson coefficient, but it is not yet clear if this is due to hadronic uncertainties [54-57] or a genuine contribution from new physics [58-61]. In FlavBit, we incorporate a $10 \%$ theoretical uncertainty (at the amplitude level) into our correlation matrix for $B^{0} \rightarrow K^{* 0} \mu^{+} \mu^{-}$observables, to account for errors arising from non-factorisable power corrections [62].

As set out in Tables 3 and 4, FlavBit can calculate the full suite of observables for $B^{0} \rightarrow K^{* 0} \mu^{+} \mu^{-}$, in six different $q^{2}$ bins over the range $1.1 \leq q^{2} / \mathrm{GeV}^{2} \leq 19.0$. These are provided by the capabilities BKstarmumu_l_m, where the lower $q^{2}$ bin edge is denoted by $l$ and the upper edge by $m$. The functions with these capabilities return a Flav_KstarMuMu_obs object (Table 4), which contains the overall branching fraction, forward-backward asymmetry and detailed angular observables $F_{\mathrm{L}}, S_{3}, S_{4}, S_{5}, S_{7}, S_{8}$ and $S_{9}$. These observables can either be extracted manually from the Flav_KstarMuMu_obs object itself, or output in full via the GAMBIT printer system [4] for later analysis.

The angular analysis of $B^{0} \rightarrow K^{* 0} \ell^{+} \ell^{-}$[63] at much lower momentum transfer $\left(q^{2} \lesssim 1 \mathrm{GeV}^{2}\right)$ can also provide strong constraints, specifically on the coefficients $C_{7}^{(\prime)}$. However, experimental analyses of $B^{0} \rightarrow K^{* 0} \mu^{+} \mu^{-}$in this regime are impacted by the assumption that the muon is massless. We therefore do not include this lower angular bin in FlavBit.

Asymmetries between $B^{0}$ and $\overline{B^{0}}$ in $B^{0} \rightarrow K^{* 0} \mu^{+} \mu^{-}$ have also been measured by the LHCb collaboration [47]. These are important for constraining the imaginary parts of a number of Wilson Coefficients.

Another observable useful for isolating the contribution of new physics, owing to its insensitivity to hadronic parameters such as form factors, is the $C P$-averaged $B \rightarrow K^{*} \mu^{+} \mu^{-}$ isospin asymmetry [64], $\frac{\mathrm{d} A_{I}}{\mathrm{~d} q^{2}} \equiv \frac{\mathrm{d} \Gamma_{B^{0} \rightarrow K^{* 0} \mu^{+} \mu^{-}} / \mathrm{d} q^{2}-\mathrm{d} \Gamma_{B^{ \pm} \rightarrow K^{* \pm} \mu^{+} \mu^{-}} / \mathrm{d} q^{2}}{\mathrm{~d} \Gamma_{B^{0} \rightarrow K^{* 0} \mu^{+} \mu^{-}} / \mathrm{d} q^{2}+\mathrm{d} \Gamma_{B^{ \pm} \rightarrow K^{* \pm} \mu^{+} \mu^{-}} / \mathrm{d} q^{2}}$.

FlavBit provides the integrated low- $q^{2}$ asymmetry, corresponding to the integral of Eq. 22 over the range $1 \leq$ $q^{2} / \mathrm{GeV}^{2} \leq 6$ (AI_BKstarmumu in Table 3 ). It also computes the zero-crossing of the asymmetry, corresponding to the $q^{2}$ value where the differential decay rates of $B^{0} \rightarrow K^{* 0} \mu^{+} \mu^{-}$ and $B^{ \pm} \rightarrow K^{* \pm} \mu^{+} \mu^{-}$are equal (AI_BKstarmumu_zero in Table 3).

The measurement of the inclusive branching fraction of $B \rightarrow X_{S} \ell^{+} \ell^{-}$is challenging from the experimental point of view, however has several theory advantages. The differential decay rate at leading order in QCD can be written as (see Ref. [65] and references therein):

$$
\begin{aligned}
& \frac{\mathrm{d} \mathcal{B}\left(B \rightarrow X_{s} \ell^{+} \ell^{-}\right)}{\mathrm{d} \hat{s}}=\mathcal{B}\left(B \rightarrow X_{c} l \bar{v}\right) \frac{\alpha^{2}}{4 \pi^{2} f(z)} \frac{\left|V_{t b} V_{t s}^{*}\right|^{2}}{\left|V_{c b}\right|^{2}} \\
& \times(1-\hat{s})^{2} \sqrt{1-\frac{4 m_{\ell}^{2}}{q^{2}}}\left\{\left(\left|C_{9}^{\text {eff }}\right|^{2}+\left|C_{10}\right|^{2}\right)(1+2 \hat{s})\right. \\
& \left.+4\left|C_{7}^{\text {eff }}\right|^{2}\left(1+\frac{2}{\hat{s}}\right)+12 \mathcal{R} e\left(C_{7}^{\text {eff }} C_{9}^{\text {eff }}\right)\right\}
\end{aligned}
$$

where $\hat{s} \equiv q^{2} / m_{b}^{2}, z=m_{c}^{2} / m_{b}^{2}$ and

$f(z)=1-8 z+8 z^{3}-z^{4}-12 z^{2} \ln z$.

The inclusive and differential branching fractions of $B \rightarrow$ $X_{s} \ell^{+} \ell^{-}$were measured at $B$ factories [66-69].

As detailed in Table 2, FlavBit computes predictions for $\mathcal{B}\left(B \rightarrow X_{s} \mu^{+} \mu^{-}\right)$, integrated over both high and low $q^{2}$ ranges (capabilities BRBXssmumu_highq2 and BRBXsmumu_lowq2). It also computes the branching fraction at high $q^{2}$ for the equivalent process with $\tau$ leptons in the final state, $\mathcal{B}\left(B \rightarrow X_{s} \tau^{+} \tau^{-}\right.$) (capability BRBXstautau_highq2).

A complementary $B \rightarrow X_{s} \ell^{+} \ell^{-}$angular observable is the forward-backward asymmetry $A_{\mathrm{FB}, B \rightarrow X_{s} \ell^{+} \ell^{-}}$, defined differentially with respect to $\hat{s}$ as

$A_{\mathrm{FB}, B \rightarrow X_{s} \ell^{+} \ell^{-}}(\hat{s}) \equiv \int_{0}^{1} \frac{\mathrm{d} \mathcal{B}(\hat{s}, z)}{\mathrm{d} \hat{s} \mathrm{~d} z}-\int_{-1}^{0} \frac{\mathrm{d} \mathcal{B}(\hat{s}, z)}{\mathrm{d} \hat{\mathrm{s}} \mathrm{d} z}$,

where $z$ is the cosine of the forward angle. FlavBit computes the $B \rightarrow X_{s} \mu^{+} \mu^{-}$integrated forward-backward asymmetry at both low and high $q^{2}$ (capabilities A_BX.smumu_highq2 and $\mathrm{A} \_B X$.smumu_lowq2), along with the zero-crossing of the asymmetry, corresponding the $q^{2}$ value for which the asymmetry vanishes (A_BXssmumu_zero). It also predicts the asymmetry of the equivalent process involving $\tau$ leptons at high $q^{2}$ (capability A_BXstautau_highq2). 
The decay $B_{s} \rightarrow \phi \mu^{+} \mu^{-}$is described by the same formalism as $B \rightarrow K^{*} \mu^{+} \mu^{-}$. However, while the latter is a self-tagging decay, i.e. the flavour of the $B$ meson at decay time can be inferred by the charge of the kaon coming from the decay of the $K^{*}(892)$, this is not the case for the $B_{s} \rightarrow \phi \mu^{+} \mu^{-}$. This implies that when averaging between $B_{s}$ and $\bar{B}_{s}$, some terms of the angular distributions (including $P_{5}^{\prime}$ ) vanish. The branching ratios of both $B_{s} \rightarrow \phi \mu^{+} \mu^{-}$ and the related decay $B^{+} \rightarrow K^{+} \mu^{+} \mu^{-}$are sensitive to BSM physics, mainly via the Wilson coefficients $C_{9}^{(\prime)}$ and $C_{10}^{(\prime)}$. The measurement of the branching fraction of $B_{s} \rightarrow \phi \mu^{+} \mu^{-}$by the $\mathrm{LHCb}$ experiment [15] is also in tension with respect to SM predictions. We do not include these channels directly in FlavBit, because to do so rigorously would require the ability to recompute model-dependent BSM contributions to theoretical uncertainties. This is a capability that we anticipate including in a future version of FlavBit.

In addition, angular measurements of the decay $B^{0} \rightarrow$ $K \pi \mu^{+} \mu^{-}$outside the $K^{*}(892)$ resonance have been recently performed [70], however we do not yet have enough knowledge of the different $K^{*}$ resonances in that region of $K \pi$ invariant mass to interpret the result in terms of Wilson coefficients [71]. For this reason, the decays $B^{0} \rightarrow K \pi \mu^{+} \mu^{-}$ outside the $K^{* 0}(892)$ are not yet implemented in FlavBit.

Lepton flavour universality in $b \rightarrow s$ transitions has also been tested by measuring the ratio $R_{K}=\frac{\mathcal{B}\left(B^{+} \rightarrow K^{+} \mu^{+} \mu^{-}\right)}{\mathcal{B}\left(B^{+} \rightarrow K^{+} e^{+} e^{-}\right)}$. A tension corresponding to $2.6 \sigma$ was observed [17]. Contrary to the anomalies in the aforementioned $b \rightarrow s \ell \ell$ transitions, the tension in $R_{K}$ cannot be explained by hadronic uncertainties. Accommodating lepton flavour non-universality within the effective Hamiltonian framework of Eq. 2 requires splitting operators $\mathcal{O}_{9}^{\left({ }^{\prime}\right)}$ and $\mathcal{O}_{10}^{\left({ }^{\prime}\right)}$ into separate effective operators for different leptons. In the context of this expanded treatment, the so-called flavour anomalies in rare decays seem to form a coherent pattern, with a reduction of about $25 \%$ observed in the muonic $C_{9}$ Wilson coefficient relative to the SM prediction. In general these scenarios are not easy to accommodate within the MSSM, although a global agreement at the $2 \sigma$ level is still possible [72]. Presently, FlavBit does not deal with violations of lepton flavour universality, so $R_{K}$ is not yet included as an observable.

\subsection{Rare purely leptonic decays}

Like its penguin counterparts $B \rightarrow X \ell^{+} \ell^{-}$, the rare leptonic decay $B_{s}^{0} \rightarrow \ell^{+} \ell^{-}$also probes the FCNC $b \rightarrow s$ transition, and is proportional to the CKM entries $V_{t b}$ and $V_{t s}$. Similarly, $B^{0} \rightarrow \ell^{+} \ell^{-}$probes $b \rightarrow d$ and is proportional to $V_{t b}$ and $V_{t d}$. These are rather clean channels from the theoretical perspective, as the main uncertainty comes only from the meson decay constant, which can be calculated in lattice QCD. The branching fraction of these decays is

$$
\begin{gathered}
\mathcal{B}\left(B_{q}^{0} \rightarrow \ell^{+} \ell^{-}\right)=\frac{G_{F}^{2} \alpha^{2}}{64 \pi^{3}} f_{B_{q}}^{2} \tau_{B_{q}} m_{B_{q}}^{3}\left|V_{t b} V_{t q}^{*}\right|^{2} \\
\times \sqrt{1-\frac{4 m_{\ell}^{2}}{m_{B_{q}}^{2}}}\left\{\left(1-\frac{4 m_{\ell}^{2}}{m_{B_{q}}^{2}}\right)\left|C_{Q 1}-C_{Q 1}^{\prime}\right|^{2}\right. \\
\left.+\left|\left(C_{Q 2}-C_{Q 2}^{\prime}\right)+2 \frac{m_{\ell}}{m_{B_{q}}}\left(C_{10}-C_{10}^{\prime}\right)\right|^{2}\right\} .
\end{gathered}
$$

Because the $B$ meson is a pseudoscalar, these decays are helicity-suppressed, in addition to the GIM suppression. Therefore, in the SM and in all lepton-flavour-universal $V \pm A$ models, the ratio of the branching fractions for different leptons is given by:

$\frac{\mathcal{B}\left(B_{q} \rightarrow \ell_{1}^{+} \ell_{1}^{-}\right)}{\mathcal{B}\left(B_{q} \rightarrow \ell_{2}^{+} \ell_{2}^{-}\right)}=\frac{m_{1}^{2}}{m_{2}^{2}}$,

where $m_{1(2)}$ is the mass of the lepton $\ell_{1(2)}$. These decays set strong constraints on models with extended Higgs sectors such as the $2 \mathrm{HDM}$, as scalar contributions would alleviate the helicity suppression. Such decays are also sensitive to new bosons with $V \pm A$ couplings (e.g. $W^{\prime}$ and $W_{R}$ ), which would modify the Wilson coefficients $C_{10}^{(\prime)}$ of the SM.

FlavBit has the capability to compute the branching fraction for $B^{0} \rightarrow \mu^{+} \mu^{-}$(Bmumu in Table 2), as well as for ( $C P$-averaged) $B_{s}$ decays to $e^{+} e^{-}$and $\mu^{+} \mu^{-}$(Bsee_untag and Bsmumu_untag). The latter can also be obtained in the MSSM and SM from FeynHiggs via the FH_Flavourobs capability (see Tables 1,5).

\subsection{Other flavour observables}

Other observables included in FlavBit are $B \rightarrow X_{s} \gamma$, the ratio $R_{\mu}=\frac{\mathcal{B}\left(K \rightarrow \mu v_{\mu}\right)}{\mathcal{B}\left(\pi \rightarrow \mu v_{\mu}\right)}$, and the meson mixing $\Delta M_{s}$.

Radiative decays of $B$ mesons are important to constrain the electromagnetic operator and the corresponding Wilson coefficients $C_{7}^{(\prime)}$. The main constraint comes from the measurement of the inclusive decay $B \rightarrow X_{s} \gamma$ [73,74]. The prediction of this branching fraction is relatively clean, and benefits from the Heavy Quark Expansion in the same way as the $B \rightarrow X_{s} \ell^{+} \ell^{-}$process.

The branching ratio can be written at leading order as

$\mathcal{B}\left(\bar{B} \rightarrow X_{s} \gamma\right)=\mathcal{B}\left(\bar{B} \rightarrow X_{c} e \bar{v}\right)_{\exp }\left|\frac{V_{t s}^{*} V_{t b}}{V_{c b}}\right|^{2} \frac{6 \alpha}{\pi C}\left|C_{7}^{\mathrm{eff}}\right|^{2}$,

where $\mathcal{B}\left(\bar{B} \rightarrow X_{c} e \bar{v}\right)_{\exp }$ is the experimentally-measured value of the branching fraction for $\bar{B} \rightarrow X_{c} e \bar{\nu}$, and

$C=\left|\frac{V_{u b}}{V_{c b}}\right|^{2} \frac{\mathcal{B}\left(\bar{B} \rightarrow X_{c} e \bar{\nu}\right)}{\mathcal{B}\left(\bar{B} \rightarrow X_{u} e \bar{\nu}\right)}$. 
Table 5 Flavour observables contained in the fh_FlavourObs structure obtained from FeynHiggs

\begin{tabular}{|c|c|}
\hline Name & Description \\
\hline Bsg_MSSM (fh_real) & Total inclusive branching fraction of $B \rightarrow X_{s} \gamma$ in the MSSM \\
\hline Bsg_SM (fh_real) & Total inclusive branching fraction of $B \rightarrow X_{s} \gamma$ in the SM \\
\hline DeltaMs_MSSM (fh_real) & $B_{s}^{0}-\overline{B_{s}^{0}}$ mass difference in the MSSM \\
\hline DeltaMs_SM(fh_real) & $B_{s}^{0}-\bar{B}_{s}^{0}$ mass difference in the SM \\
\hline Bsmumu_MSSM (fh_real) & Branching fraction of $B_{s}^{0} \rightarrow \mu^{+} \mu^{-}$in the MSSM \\
\hline Bsmumu_SM (fh_real) & Branching fraction of $B_{s}^{0} \rightarrow \mu^{+} \mu^{-}$in the SM \\
\hline
\end{tabular}

This measurement sets constraints on the charged Higgs mass and couplings of the 2HDM [75-78]. In addition, these measurements constrain models with additional neutral gauge bosons such as the $Z^{\prime}$ [35]. FlavBit implements this observable as bsgamma (Table 2), and within the FH_FlavourObs capability (see Tables 1,5$).^{7}$

The exclusive decays $B \rightarrow K^{*} \gamma$ and $B_{s} \rightarrow \phi \gamma$ also constrain the coefficients $C_{7}^{(\prime)}$, but their impact is not yet competitive with the inclusive one. However, the inclusive decays can only constrain the sum of $\left|C_{7}\right|^{2}$ and $\left|C_{7}^{\prime}\right|^{2}$. The best constraint on the right-handed current $C_{7}^{\prime}$ contribution presently comes from the angular analysis of $B^{0} \rightarrow K^{* 0} \ell^{+} \ell^{-}$at low $q^{2}$ (see Sect. 4.3). In FlavBit, we provide the $C P$-averaged isospin asymmetry of $B \rightarrow K^{*} \gamma$ decays [80],

$\Delta_{0} \equiv \frac{\Gamma\left(\bar{B}^{0} \rightarrow \bar{K}^{* 0} \gamma\right)-\Gamma\left(B^{ \pm} \rightarrow K^{* \pm} \gamma\right)}{\Gamma\left(\bar{B}^{0} \rightarrow \bar{K}^{* 0} \gamma\right)+\Gamma\left(B^{ \pm} \rightarrow K^{* \pm} \gamma\right)}$,

as a calculable observable, as it can receive contributions from charged Higgs bosons and any other new fields with similar quantum numbers (such as charginos in supersymmetry) [81]. The predicted asymmetry can be accessed via capability del tao (Table 2).

The leptonic decays of $K$ and $\pi$ mesons are also sensitive to the existence of charged Higgs bosons [82]. FlavBit computes the ratio [83]

$$
\begin{aligned}
R_{\mu}= & \frac{\mathcal{B}\left(K \rightarrow \mu v_{\mu}\right)}{\mathcal{B}\left(\pi \rightarrow \mu v_{\mu}\right)} \\
= & \left(1+\delta_{\mathrm{em}}\right) \frac{\tau_{K}}{\tau_{\pi}}\left|\frac{V_{u s}}{V_{u d}}\right|^{2} \frac{f_{K}^{2}}{f_{\pi}^{2}} \frac{m_{K}}{m_{\pi}}\left(\frac{1-m_{\ell}^{2} / m_{K}^{2}}{1-m_{\ell}^{2} / m_{\pi}^{2}}\right)^{2} \\
& \times\left[1-\frac{m_{K^{+}}^{2}}{M_{H^{+}}^{2}}\left(1-\frac{m_{d}}{m_{s}}\right) \frac{\tan ^{2} \beta}{1+\epsilon_{0} \tan \beta}\right]^{2},
\end{aligned}
$$

which has a smaller theoretical uncertainty than the individual decays. Here $\delta_{\mathrm{em}}=0.0070 \pm 0.0035$ is a long-distance

\footnotetext{
${ }_{7}$ Note that the branching fraction of $B \rightarrow X_{s} \gamma$ is ill-defined for $E_{\gamma} \rightarrow 0$, due to the IR divergence associated with soft photon emission. Although the adopted cutoff on $E_{\gamma}$ is unspecified in FeynHiggs, $\mathcal{B}\left(B \rightarrow X_{s} \gamma\right)$ here appears to follow the definition of 'total' advocated in Ref. [79], with $E_{\gamma}>m_{b} / 10 \sim 0.4 \mathrm{GeV}$.
}

electromagnetic correction factor. We also consider the quantity $R_{\mu 23}[83]$,

$$
\begin{aligned}
R_{\mu 23} & =\left|\frac{V_{u s}\left(K_{\ell 2}\right)}{V_{u s}\left(K_{\ell 3}\right)} \times \frac{V_{u d}\left(0^{+} \rightarrow 0^{+}\right)}{V_{u d}\left(\pi_{\ell 2}\right)}\right| \\
& =\left|1-\frac{m_{K^{+}}^{2}}{M_{H^{+}}^{2}}\left(1-\frac{m_{d}}{m_{s}}\right) \frac{\tan ^{2} \beta}{1+\epsilon_{0} \tan \beta}\right|,
\end{aligned}
$$

where $\ell_{i}$ refers to leptonic decays with $i$ particles in the final state, and $0^{+} \rightarrow 0^{+}$corresponds to nuclear beta decay. These are provided by capabilities Rmu and Rmu23, respectively, and the relevant functions are detailed in Table 1.

It is well known that neutral meson systems are characterised by a rich phenomenology. In general, eigenstates of flavour are not eigenstates of mass, causing neutral mesons to oscillate. The parameters governing oscillations are the difference in mass between the heavy and light eigenstates $\Delta M=M_{H}-M_{L}$ and the difference in their decay widths $\Delta \Gamma=\Gamma_{H}-\Gamma_{L}$. While in the neutral kaon system the difference in lifetime is very large, so we denote the two states 'short' $\left(K_{S}^{0}\right)$ and 'long' $\left(K_{L}^{0}\right)$, in the neutral $B$ system $\Delta \Gamma \ll \Delta M$, so it is more suitable to call them 'heavy' and 'light'. The oscillation frequency is related to the difference in mass $\Delta M_{q}$, which for the neutral $B$ meson is

$\Delta M_{q}=\frac{G_{F}^{2}}{6 \pi^{2}} \eta_{B} m_{B_{q}}\left(\hat{B}_{q} f_{B_{q}}^{2}\right) M_{W}^{2} S_{0}\left(x_{t}\right)\left|V_{t q}\right|^{2}$

where $\hat{B}_{q}$ is the renormalisation-group-invariant parameter, $f_{B_{q}}$ is the $B_{q}$ decay constant and $S_{0}\left(x_{t}\right)$ is a simple function of the top mass. The hadronic parameter $f_{B_{q}}$ is the same factor that appears in the branching fraction of $B_{q} \rightarrow \ell^{+} \ell^{-}$decays (Eq. 26). The branching fractions and mass differences are therefore related as [84]

$\frac{\mathcal{B}\left(B_{s}^{0} \rightarrow \ell^{+} \ell^{-}\right)}{\mathcal{B}\left(B^{0} \rightarrow \ell^{+} \ell^{-}\right)}=\frac{\hat{B}_{s}}{\hat{B}_{d}} \frac{\tau\left(B_{s}^{0}\right)}{\tau\left(B^{0}\right)} \frac{\Delta M_{s}}{\Delta M_{d}}$.

In FlavBit, $\Delta M_{S}$ can be obtained in either the SM or MSSM, via the FH_Flavourobs capability (see Tables 1, 5). 
Table 6 Parameters of a single experimental entry in the FlavBit YAML database

\begin{tabular}{|c|c|}
\hline Name & Description \\
\hline name & Unique name of a given measurement \\
\hline islimit & $\begin{array}{l}\text { Flag that indicates if the measurement is } \\
\text { in the form of an upper limit (true) or } \\
\text { a measurement (false) }\end{array}$ \\
\hline exp_value & $\begin{array}{l}\text { The experimental measurement (if } \\
\text { islimit }=\text { false) or limit (if } \\
\text { islimit }=\text { true) }\end{array}$ \\
\hline exp_stat_error & $\begin{array}{l}1 \sigma \text { uncorrelated statistical uncertainty on } \\
\text { the experimental measurement or limit }\end{array}$ \\
\hline exp_sys_error & $\begin{array}{l}1 \sigma \text { uncorrelated systematic uncertainty on } \\
\text { the experimental measurement or limit }\end{array}$ \\
\hline exp_source & $\begin{array}{l}\text { The source of the experimental value and } \\
\text { uncertainties }\end{array}$ \\
\hline th_error & $1 \sigma$ uncorrelated theoretical uncertainty \\
\hline th_error_type & $\begin{array}{l}\text { Flag indicating whether the theory error is } \\
\text { multiplicative }(\mathrm{M}) \text { or additive }(\mathrm{A}) \text {. }\end{array}$ \\
\hline th_source & The source of the theoretical uncertainty \\
\hline \multirow[t]{5}{*}{ correlation } & $\begin{array}{l}\text { Sub-section with correlations of the } \\
\text { experimental measurement/limit to other } \\
\text { experimental measurements/limits: }\end{array}$ \\
\hline & $\begin{array}{l}\text { name Name of another } \\
\text { measurement with which this one is } \\
\text { correlated }\end{array}$ \\
\hline & $\begin{array}{l}\text { value Correlation matrix entry } \\
\text { relating the two measurements }\end{array}$ \\
\hline & $\begin{array}{l}\text { name Name of a third measurement } \\
\text { with which this one is correlated }\end{array}$ \\
\hline & value etc \\
\hline
\end{tabular}

\section{Likelihoods}

After calculating the observables described in Sect. 4, FlavBit can be used to compute likelihoods based on a comparison of the predictions with current experimental measurements.

The experimental results and theoretical errors are stored in a YAML database. Taking the branching fraction of $B_{s} \rightarrow$ $\mu^{+} \mu^{-}$as an example, the FlavBit database entry is

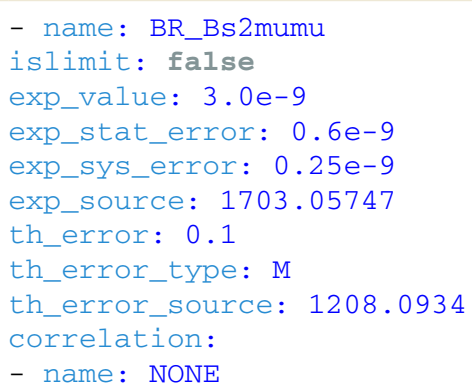

The individual fields available in such entries are described in detail in Table 6 . Note in particular that the theory error may be given either as a fraction, as in this example, or as an absolute value. The Flav_reader object is responsible for reading the experimental results and theoretical errors, and calculating the resulting covariance matrix. Table 7 describes its specific functions.

We consider correlated theoretical and experimental uncertainties separately, building two covariance matrices and assuming linear correlations for both. In the case of asymmetric uncertainties, we symmetrise the errors by taking the mean of the upper and lower uncertainties. FlavBit constructs the experimental covariance matrix directly from the exp_stat_error, exp_sys_error and correlation entries in its YAML database (Table 6 and example above). It takes the th_error entries in the YAML database and uses them to populate the diagonal of the theory covariance matrix. It determines the off-diagonal terms on a case-bycase basis in each likelihood function, in order to make it possible for different likelihood functions to adjust the correlations according to whether different nuisance parameters are scanned over directly, or should be included via the correlation matrix. ${ }^{8}$

FlavBit builds the full covariance matrix by summing the experimental and theoretical covariance matrices. If an observable and its measurements are uncorrelated with other observables, the resulting uncertainty then becomes simply the sum in quadrature of the theoretical and experimental errors.

We determine likelihoods for flavour observables under the assumption of correlated Gaussian errors and Wilks' Theorem, taking (twice) the final log-likelihood to be $\chi^{2}$ distributed. This gives

$\log \mathcal{L}=-\frac{1}{2} \chi^{2}=-\frac{1}{2} \sum_{i, j=1}^{N}\left(y_{i}-x_{i}\right) V_{i j}^{-1}\left(y_{j}-x_{j}\right)$,

where $x_{i}$ is the experimental measurement of the $i$ th observable, $y_{i}$ is the $i$ th theory prediction and $V^{-1}$ is the inverse of the full covariance matrix.

\footnotetext{
${ }^{8}$ Users of FlavBit should be aware of a potential pitfall arising from this arrangement. The theory uncertainties and correlations that we include in the current release and describe in this paper already incorporate uncertainties on input parameters such as form factors, decay constants, SM masses and couplings, and in particular, CKM matrix entries. The SM masses and couplings are sufficiently well constrained that any error term dominated by them can be safely neglected, and generally is in FlavBit, seeing as they can be easily varied within GAMBIT as nuisance parameters. On the other hand, CKM elements are substantial and dominant contributors to the error budget of some processes. The current likelihoods in FlavBit should therefore not be employed in any scan where CKM elements are varied as nuisance parameters, without first carefully considering which likelihood terms already include their impact, and either removing those observables from the fit, or reducing the theory errors accordingly.
} 
Table 7 Important methods of the FlavBit Flav_reader class. Here str is an alias for std: : string, $n$ is the number of measurements so far read in by the Flav_reader instance, and matrix $(x, y)$ is an $x \times y$ boost: :numeric: :ublas: :matrix<double>

\begin{tabular}{|c|c|}
\hline Name & Description \\
\hline int read_yaml(str name) & Reads an entire YAML database file name into memory \\
\hline $\begin{array}{l}\text { void read_yaml_measurement(str name, str } \\
\text { measurement_name) }\end{array}$ & $\begin{array}{l}\text { Extracts a single measurement measurement_name from the YAML } \\
\text { database file name }\end{array}$ \\
\hline void debug_mode(bool debug) & $\begin{array}{l}\text { Turns on }(\text { debug }=\text { true) or off (debug }=\text { false) printing of all } \\
\text { parameters }\end{array}$ \\
\hline void create_global_corr() & Constructs a total correlation matrix from all measurements read in \\
\hline void print_corr_matrix() & Prints the constructed correlation matrix \\
\hline void print_cov_matrix() & Prints the corresponding covariance matrix \\
\hline void print_cov_inv_matrix() & Prints the inverse of the covariance matrix \\
\hline matrix $(n, n)$ get_cov() & $\begin{array}{l}\text { Returns the experimental covariance matrix covering all measurements } \\
\text { read in }\end{array}$ \\
\hline matrix $(n, 1)$ get_exp_value() & Returns the central experimental values for all measurements read in \\
\hline $\operatorname{matrix}(n, 1)$ get_th_err() & $\begin{array}{l}\text { Returns the central (uncorrelated) theory error for each of the } \\
\text { measurements read in }\end{array}$ \\
\hline
\end{tabular}

FlavBit contains five different likelihood functions. These correspond to different likelihood classes within which observables might be correlated.

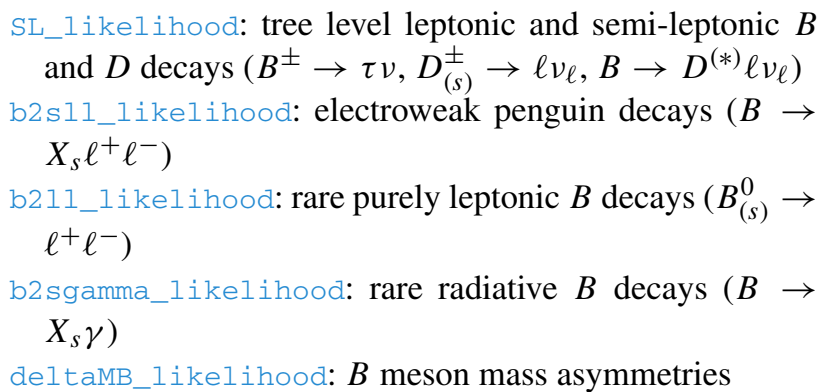

The likelihood functions, their capabilities and dependencies are given in Table 8. In the following subsections, we give details of the experimental data included in each.

\subsection{Tree-level leptonic and semi-leptonic likelihood}

We take the branching fractions of the decays $B \rightarrow D^{(*)} \ell v_{\ell}$ from the PDG [28], which combines results from many experiments but is dominated by the contributions from $\mathrm{BaBar}$ $[85,86]$ and Belle $[87,88]$.

BaBar $[19,20]$ and Belle $[21,89,90]$ also recently measured the ratios $R_{D^{(*)}} \equiv \mathcal{B}\left(B \rightarrow D^{(*)} \tau v_{\tau}\right) / \mathcal{B}(B \rightarrow$ $\left.D^{(*)} \ell v_{\ell}\right)$. LHCb also measured $R_{D^{*}}$ for the muonic final state [16]. The average of these measurements, assuming lepton flavour universality between muons and electrons, has been computed by the HFAG collaboration $[91,92]$ and is included in FlavBit:

$$
R_{D}=0.403 \pm 0.040 \pm 0.024,
$$

$R_{D^{*}}=0.310 \pm 0.015 \pm 0.008$.

Compared to the SM predictions of $R_{D}=0.300 \pm 0.008$ [93] and $R_{D^{*}}=0.252 \pm 0.003$ [94], a total discrepancy of about $4 \sigma$ is observed. We take the experimental correlation between $R_{D}$ and $R_{D^{*}}$, arising from common systematics in the measurements, from Ref. [91]. The theory uncertainties are considered uncorrelated; we take these from Refs. $[95,96]$.

In addition to $R_{D}$ and $R_{D^{*}}$, we also explicitly include in the likelihood the decays $B \rightarrow D^{(*)} \mu \nu$, adopting the experimental values from the PDG [28]. Taken with $R_{D}$ and $R_{D^{*}}$, this set of four likelihood terms constitutes a complete basis for the models of lepton non-universality. The theory errors for the $B \rightarrow D^{(*)} \mu \nu$ branching fractions are dominated by form factors $[29,93]$. Performing a detailed error analysis with Superlso gives a theoretical uncertainty of $9 \%$ for $B \rightarrow D \mu \nu$ and $11 \%$ for $B \rightarrow D^{*} \mu \nu$.

Experiments have not measured any correlation between the muonic and tauonic modes of the decays contributing to $R_{D^{(*)}}$. However, the theory systematics are strongly correlated; in our analysis with Superlso, we find anticorrelations at the level of $55 \%$ for $B \rightarrow D \mu \nu$ and $R_{D}$, and $62 \%$ for $B \rightarrow D^{(*)} \mu \nu$ and $R_{D^{*}}$. These data are all included in the FlavBit likelihood.

For $B^{ \pm} \rightarrow \ell v_{\ell}$, FlavBit uses experimental measurements from the PDG [28],

$\mathcal{B}\left(B^{+} \rightarrow \tau^{+} v_{\mu}\right)=(1.09 \pm 0.24) \times 10^{-4}$.

This average is dominated by results from the $\mathrm{BaBar}[97,98]$ and Belle $[99,100]$ experiments, and is in agreement with the SM. We take this measurement to be uncorrelated with all other measurements. The dominant theoretical uncertainty comes from the CKM element $V_{u b}$. The present uncertainty 
Table 8 Likelihood capabilities of FlavBit. All measurement functions (capabilities ending in _M) return experimental and theoretical central values, as well as experimental and theoretical covariance matrices

\begin{tabular}{|c|c|c|}
\hline Capability & Function (return type): brief description & Dependencies \\
\hline SL_M & $\begin{array}{l}\text { SL_measurements } \\
\text { (predictions_measurements_covariances): } \\
\text { Tree-level leptonic and semi-leptonic decay predictions, } \\
\text { measurements and covariances }\end{array}$ & $\begin{array}{l}\text { RD } \\
\text { RDstar } \\
\text { BDmunu } \\
\text { BDstarmunu } \\
\text { Btaunu } \\
\text { Dstaunu } \\
\text { Dsmunu } \\
\text { Dmunu }\end{array}$ \\
\hline SL_LL & $\begin{array}{l}\text { SL_likelihood (double): } \\
\text { Log-likelihood for tree-level leptonic and semi-leptonic } \\
\text { decays }\end{array}$ & SL_M \\
\hline b2sl1_M & $\begin{array}{l}\text { b2,sl1_measurements } \\
\text { (predictions_measurements_covariances): } \\
\text { Electroweak penguin decay predictions, measurements and } \\
\text { covariances }\end{array}$ & $\begin{array}{l}\text { BKstarmumu_11_25 } \\
\text { BKstarmumu_25_40 } \\
\text { BKstarmumu_40_60 } \\
\text { BKstarmumu_60_80 } \\
\text { BKstarmumu_15_17 } \\
\text { BKstarmumu_17_19 }\end{array}$ \\
\hline b2sl1_LL & $\begin{array}{l}\text { b2sll_likelihood (double): } \\
\text { Log-likelihood for electroweak penguin decays, including } \\
\text { angular observables }\end{array}$ & $\mathrm{b} 2 \mathrm{~s} 11 \_\mathrm{M}$ \\
\hline b211_M & $\begin{array}{l}\text { b211_measurements } \\
\text { (predictions_measurements_covariances): } \\
\text { Rare purely leptonic decay predictions, measurements and } \\
\text { covariances }\end{array}$ & $\begin{array}{l}\text { Bsmumu_untag } \\
\text { Bmumu }\end{array}$ \\
\hline b211_LL & $\begin{array}{l}\text { b211_likelihood (double): } \\
\text { Log-likelihood for rare purely leptonic decays }\end{array}$ & b211_M \\
\hline b2sgamma_LL & $\begin{array}{l}\text { b2sgamma_likelihood (double): } \\
\text { Log-likelihood for the branching fraction of } B \rightarrow X_{s} \gamma\end{array}$ & bsgamma \\
\hline deltaMB_LL & $\begin{array}{l}\text { deltaMB_likelihood (double): } \\
\text { Log-likelihood for } B \text { meson mass asymmetries }\end{array}$ & deltaMs \\
\hline
\end{tabular}

on this element is $9.5 \%$ [28], giving an overall theoretical uncertainty of $19 \%$.

For the branching fractions of the $D_{(s)}^{ \pm}$decays $D^{ \pm} \rightarrow$ $\mu v_{\mu}, D_{s}^{ \pm} \rightarrow \tau v_{\tau}$ and $D_{s}^{ \pm} \rightarrow \mu v_{\mu}$, we adopt the experimental values of the PDG [28]. (FlavBit does not include $D^{ \pm} \rightarrow \tau v_{\tau}$ as an observable, as its decay branching fraction has not yet been measured.) The theory errors on the $D_{(s)}^{ \pm}$decays are dominated by the knowledge of the decay constant of the corresponding charmed mesons, $f_{D}$ and $f_{D_{s}}$. This leads to a theoretical uncertainty on the branching fractions of $3 \%$ for $D^{ \pm}$decays and $2 \%$ for $D_{s}^{ \pm}$decays [28].

As shown in Table 8, FlavBit collects together into $S L_{-}{ }^{\mathrm{M}}$ the measured values, experimental correlations, theoretical predictions and theory uncertainties for $B^{ \pm} \rightarrow \ell v_{\ell}$, the four $B \rightarrow D^{(*)} \ell v_{\ell}$ observables, and the three $D_{(s)}^{ \pm}$decays. This fills the only dependency of the final tree-level leptonic and semi-leptonic likelihood, which can be accessed via capability $S L \_L L$.

\subsection{Electroweak penguin likelihood}

The electroweak penguin likelihood in FlavBit is calculated using the angular observables of the $B^{0} \rightarrow K^{* 0} \mu^{+} \mu^{-}$decay, as measured by $\mathrm{LHCb}$ [47] in dimuon invariant mass squared bins of $(1.1,2.5),(2.5,4),(4,6),(6,8),(15,17)$ and $(17$, 19) $\mathrm{GeV}^{2}$. The bin $(11,12.5) \mathrm{GeV}^{2}$ cannot be used in the likelihood, as the relative phase between the charmonium resonances in this bin and the non-resonant decay is not currently known. We do not implement the measurements of Belle [23], as their contribution to the likelihood is negligible compared 
to the LHCb measurement. ATLAS and CMS have also very recently presented preliminary Run I measurements of the $B^{0} \rightarrow K^{* 0} \mu^{+} \mu^{-}$angular observables [101,102]; these data will be included in a future release of FlavBit.

For each $q^{2}$ bin, the FlavBit likelihood includes components arising from $\mathrm{FL}, \mathrm{S} 3, \mathrm{~S} 4, \mathrm{~S} 5, \mathrm{AFB}, \mathrm{S} 7, \mathrm{~S} 8$ and $\mathrm{S} 9$. It accounts for experimental correlations between these measurements within each bin, but assumes that measurements are not correlated across $q^{2}$ bins, as the uncertainty is dominated by the statistical component. The full correlation matrices within each bin are available publicly from LHCb [47] and included in the FlavBit YAML database. We include theory-induced correlated uncertainties between different angular observables for the same $q^{2}$ range from Refs. [62,103].

The branching fractions for $B^{0} \rightarrow K^{* 0} \mu^{+} \mu^{-}$decays are not part of the electroweak penguin likelihood in FlavBit 1.0.0, but are slated for inclusion in a future version, following the next update from LHCb. The isospin asymmetry of the $B^{0} \rightarrow K^{* 0} \mu^{+} \mu^{-}$decay is non-trivially correlated with the angular observables, so we also do not include the corresponding observables (AI_BKstarmumu and AI_BKstarmumu_zero in Table 3) in the likelihood function.

Predictions of the branching fractions and forwardbackward asymmetries of the inclusive decays $B \rightarrow$ $X_{s} \mu^{+} \mu^{-}$and $B \rightarrow X_{s} \tau^{+} \tau^{-}$, corresponding to the last 7 observables of Table 2, have lower theoretical uncertainties than those of $B^{0} \rightarrow K^{* 0} \mu^{+} \mu^{-}$. They are however not included in the FlavBit electroweak penguin likelihood, as they provide little additional constraining power when $B \rightarrow X_{s} \gamma$ is already included in a fit - and only $B \rightarrow X_{s} \ell^{+} \ell^{-}$(where $\ell$ does not distinguish between $e$ and $\mu$ ) and its forward-backward asymmetry have been measured by BaBar and Belle [66-69], with higher uncertainties than measurements of the exclusive modes. We expect to include likelihoods for these observables in a future revision of FlavBit.

FlavBit reads the experimental measurements and correlations, collects them together with the theoretical predictions and uncertainties, and publishes them to the rest of GAMBIT under the capability b2s11_M. FlavBit then uses the measurements and correlations to compute the electroweak penguin decay likelihood, which is assigned capability b2sII_LI. See Table 8 for more details.

\subsection{Rare purely leptonic likelihood}

Experimentally, only the decays with muons in the final state have been observed, and therefore give the strongest constraints. For $B_{s}^{0} \rightarrow \mu^{+} \mu^{-}$, we adopt the latest result from LHCb [104],

$$
\mathcal{B}\left(B_{s}^{0} \rightarrow \mu^{+} \mu^{-}\right)=\left(3.0 \pm 0.6_{-0.2}^{+0.3}\right) \times 10^{-9} .
$$

For $B^{0} \rightarrow \mu^{+} \mu^{-}$, we take the results of Ref. [105], which combines the measurements of the LHCb [106] and CMS experiments [107],

$\mathcal{B}\left(B^{0} \rightarrow \mu^{+} \mu^{-}\right)=\left(3.9_{-1.4}^{+1.6}\right) \times 10^{-10}$.

Experimental correlations between the two decays are negligible [104].

Although the ATLAS collaboration have also recently measured these two branching fractions [108], they do not yet report a $3 \sigma$ evidence for these decays. We thus do not include the ATLAS result in FlavBit at this stage. The similar decays $B_{(s)}^{0} \rightarrow e^{+} e^{-}$and $B_{(s)}^{0} \rightarrow \tau^{+} \tau^{-}$have not been measured to date. Only weak upper limits exists in these cases [109111], which are currently much less constraining for models of new physics than the muon channels; we therefore do not include them in the FlavBit likelihood.

From the theoretical point of view, $B_{(s)}^{0} \rightarrow \mu^{+} \mu^{-}$decays are rather clean. The theory uncertainty is $10 \%$, and is dominated by the knowledge of the meson decay constant $f_{B_{s}}$ [112]. This is far smaller than the experimental uncertainty, and therefore has little impact. We also neglect corresponding correlations in the theoretical uncertainties associated with the two decays.

FlavBit reads the experimental measurements and theory errors, collects them together with the theoretical predictions, and publishes them to the rest of GAMBIT as b211_M. It then computes the rare purely leptonic decay likelihood from the measurements and uncertainties, and labels it with capability b211_LL. Table 8 gives full details.

\subsection{Rare radiative $B$ decay likelihood}

FlavBit includes the average [78] of the measurements of $B \rightarrow X_{s} \gamma$ from BaBar [113-115] and Belle [116,117] for $E_{\gamma}>1.6 \mathrm{GeV}$,

$\mathcal{B}\left(B \rightarrow X_{s} \gamma\right)=(3.27 \pm 0.14) \times 10^{-4}$.

We adopt a theoretical uncertainty of 7\%, coming partly from non-perturbative effects [77,118]. The corresponding likelihood has capability b2sgamma_LL (Table 8), and consists of a direct call to the standard GAMBIT Gaussian likelihood [4]. Note that in general the theoretical calculation from Superlso should be preferred over the corresponding quantity from FeynHiggs as input to this likelihood, as the cut employed on the photon energy in SI_bsgamma $\left(E_{\gamma}>1.6 \mathrm{GeV}-\right.$ see Table 2$)$ is correctly matched to the cut applied in the experimental analysis.

The experimental correlation between $\mathcal{B}\left(B \rightarrow X_{s} \gamma\right)$ and the isospin asymmetry of $B \rightarrow K^{*} \gamma$ is not known, though it is expected to be non-negligible given that the event selections overlap. Because the inclusive branching ratio of 
$B \rightarrow X_{s} \gamma$ has a smaller theoretical uncertainty, we include $\mathcal{B}\left(B \rightarrow X_{s} \gamma\right)$ but not $\Delta_{0}$ in the likelihood function.

\section{$5.5 B$ meson mass asymmetry likelihood}

The parameters $\Delta M_{s}$ and $\Delta M_{d}$ have been precisely measured [91]:

$$
\begin{aligned}
\Delta M_{d} & =0.5064 \pm 0.0019 \mathrm{ps}^{-1}, \\
\Delta M_{s} & =17.757 \pm 0.021 \mathrm{ps}^{-1} .
\end{aligned}
$$

The measurement of $\Delta M_{d}$ is the average of the results from the DELPHI, ALEPH, L3, OPAL, CDF, D0, BaBar, Belle and $\mathrm{LHCb}$ experiments, while the $\Delta M_{S}$ value is the average of the results from the $\mathrm{CDF}$ and $\mathrm{LHCb}$ experiments. The sensitivity of these observables is diluted by the theory uncertainty, which is essentially the same for both SM and BSM predictions, as it is dominated by lattice calculations of non-perturbative effects and the uncertainty on the $B$ decay constant $f_{B}$. The total theoretical uncertainty on $\Delta M_{S}$, for example, is currently $15 \%$ [119].

At present, FlavBit can predict only $\Delta M_{S}$ (Table 1), so the $B$ meson mass asymmetry likelihood simply compares this prediction to Eq. 43, using a theoretical error of $15 \%$ and the standard GAMBIT Gaussian likelihood function [4]. This likelihood is available via the capability deltaMB_LL (Table 8).

\subsection{Other observables}

The $R_{\mu 23}$ average is dominated by the KLOE [120] and NA62 [121] experiments. While both $R_{\mu}$ and $R_{\mu 23}$ are implemented as observables in FlavBit, they are not included in the likelihood. For several BSM models, such as the 2HDM, they add negligible additional constraints, particularly when the decay $B^{ \pm} \rightarrow \tau \nu_{\tau}$ is included in the likelihood via SL_likelihood.

\section{Examples}

Basic examples of how to use FlavBit in a GAMBIT BSM global fit can be found in any of the canonical GAMBIT SUSY examples in the yaml_files directory: CMSSM.yam1, NUHM1 • yaml, NUHM2 . yaml or MSSM7 . yaml [4, 10, 11]. In this section, we go through a number of flavour-specific examples, ranging from flavour-only supersymmetric and effective field theory scans with GAMBIT, to an example of how to use FlavBit in standalone mode.
Table 9 CMSSM parameters varied in the example fit, along with their associated ranges and prior types. The "hybrid" prior on $A_{0}$ is logarithmic for $\left|A_{0}\right|>100 \mathrm{GeV}$ and flat for $\left|A_{0}\right|<100 \mathrm{GeV}$

\begin{tabular}{llll}
\hline Parameter & Minimum & Maximum & Prior \\
\hline$m_{0}$ & $50 \mathrm{GeV}$ & $7 \mathrm{TeV}$ & $\log$ \\
$m_{\frac{1}{2}}$ & $50 \mathrm{GeV}$ & $5 \mathrm{TeV}$ & $\log$ \\
$A_{0}$ & $-10 \mathrm{TeV}$ & $10 \mathrm{TeV}$ & hybrid \\
$\tan \beta$ & 3 & 70 & flat \\
$\alpha_{s}^{\overline{M S}}\left(m_{Z}\right)$ & 0.1167 & 0.1203 & flat \\
$m_{t, \text { pole }}$ & 171.06 & 175.62 & flat \\
\hline
\end{tabular}

\subsection{Supersymmetric scan}

It is often instructive to consider the impacts of restricted classes of observables on broader global fits. In yaml_files/FlavBit_CMSSM.yaml, we give an example of a Constrained MSSM (CMSSM) fit focussing specifically on observables and likelihoods from FlavBit. This scan varies three dimensionful Lagrangian parameters defined at the GUT scale (the trilinear coupling $A_{0}$, the universal scalar mass $m_{0}$ and the universal fermion mass $m_{\frac{1}{2}}$ ), the dimensionless ratio of Higgs VEVs at the weak scale $(\tan \beta)$, and two SM nuisance parameters $\left(\alpha_{s}\right.$ and $\left.m_{t}\right)$. The parameters and ranges are shown in Table 9.

In this example scan, we include the FlavBit rare leptonic and semileptonic (SL_LL), electroweak penguin (b2s $\left.11 \_L L\right)$, rare purely leptonic (b211_LL) and rare radiative likelihoods (b2sgamma_LL). In the interests of speed, numerical stability and comparability to the main CMSSM results presented in Ref. [10], we do not include the prediction of $\Delta M_{S}$ from FeynHiggs nor the resulting $B$ mass asymmetry likelihood (deltamB_LL). We employ nuisance likelihoods from PrecisionBit [14] to constrain $\alpha_{s}$ and $m_{t}$.

We focus specifically on the frequentist profile likelihood in this scan, and therefore employ differential evolution to sample the parameter space, as implemented in Diver [8]. Consistent with Ref. [10], we choose a population of 19200 and a convergence threshold of $10^{-5}$. Although the profile likelihood is in principle independent of the chosen sampling method and prior, in practice these have an impact on the sampling efficiency and the ability of a scan to uncover more isolated likelihood modes $[8,122,123]$. Our scans employ effectively logarithmic priors on the dimensionful BSM parameters, and flat priors on all other parameters. The SM parameters are sufficiently well constrained that the prior is irrelevant. We discuss the impact of the sampling prior on the BSM parameters below.

The resulting scan took approximately $15 \mathrm{~min}$ to run on 1200 CPU cores, and produced 1.1 million likelihood samples. 

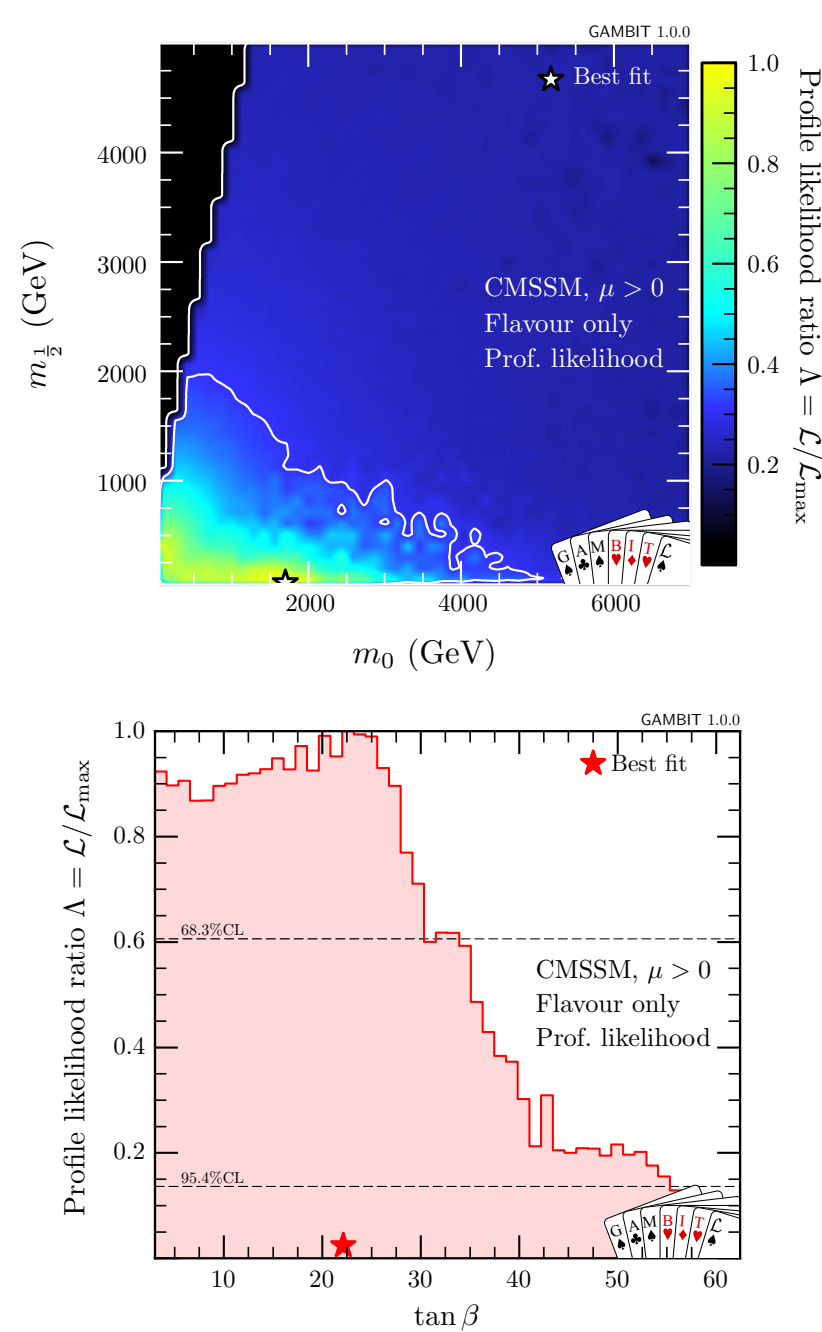

Fig. 1 2D (upper) and 1D (lower) profile likelihoods of Lagrangian parameters $m_{0}, m_{1 / 2}$ and $\tan \beta$ in a CMSSM fit including flavour and nuisance likelihoods only. Stars identify the best fit, and contours indicate 1 and $2 \sigma$ confidence regions. The jagged edge of the $2 \sigma$ contour at low $m_{0}$ and large $m_{1 / 2}$ is a plotting artefact, caused by interaction of the binning required for plotting and the abruptness of the dropoff of the likelihood in this region (due to the requirement that the lightest supersymmetric particle be a neutralino)

The results are shown in Fig. 1, in terms of the 2D profile likelihood of the sparticle masses $m_{0}$ and $m_{\frac{1}{2}}$, and the 1D profile likelihood of $\tan \beta$. The flavour likelihoods have the most impact at large $\tan \beta$, as has been extensively pointed out in the literature (e.g. $[72,124]$ ). The $2 \mathrm{D}$ figure shows a weak preference (at the 1-2 $\sigma$ level) for lower sparticle masses. At first glance this may seem surprising, given the lack of hints for SUSY, the fact that the likelihood at large $m_{0}$ and $m_{\frac{1}{2}}$ essentially recovers the SM result, and the resulting tendency of $b \rightarrow s \gamma$ to drive SUSY fits to larger masses to avoid spoiling the good agreement between the SM prediction and the observed value of $\mathcal{B}\left(B \rightarrow X_{s} \gamma\right)$. Indeed, the likelihood improvement at low mass is driven entirely by the angular analysis of $B^{0} \rightarrow K^{*} \mu^{+} \mu^{-}$decays, with the fit attempting to account for the deviation from the SM prediction in this channel by making the new states light and boosting the (generally small) SUSY contributions as much as possible. This effect is rather small, providing an improvement in the likelihood contribution from $B^{0} \rightarrow K^{*} \mu^{+} \mu^{-}$ (b2sll_likelihood) of $\Delta \ln \mathcal{L}=3.4$ relative to the $\mathrm{SM}$. This improvement is mostly counteracted by a corresponding decrease of $\Delta \ln \mathcal{L}=-2.0$ in the likelihood associated with $\mathcal{B}\left(B \rightarrow X_{s} \gamma\right)$ (b2sgamma_likelihood).

\subsection{Wilson coefficient fit}

As a more advanced example, we carry out a joint fit to the real parts of the $C_{7}, C_{9}$ and $C_{10}$ effective couplings of Eq. 2, expressed in terms of offsets from their SM values $\Delta C_{i} \equiv$ $C_{i}-C_{i, \mathrm{SM}}$. The YAML file for this scan can be found at yaml_files/WC.yaml.

In this example, we use the electroweak penguin likelihood (b2sll_likelihood), the rare purely leptonic decay likelihood (b2l1_likelihood) and the rare radiative decay likelihood (b2sgamma_likelihood). The other two likelihood functions available in FlavBit (based on the $B$ meson mass asymmetry and tree-level leptonic and semi-leptonic decays) have no dependence on the three Wilson coefficients that we vary. We also scan over the $\overline{M S} b$ quark mass and the strong coupling as nuisance parameters, computing associated nuisance likelihoods with PrecisionBit [14]. We sample the parameter space with nested sampling [125,126], using 20000 live points and a tolerance of 0.1 ; see Ref. [8] for details of the scanning setup and sampling algorithm.

The results of this scan are shown in Fig. 2. Here we show both Bayesian posterior probabilities (lower left panels) and frequentist profile likelihoods (upper right panels), which are in rather close agreement. The small offset between the peaks of the posterior and the profile likelihood in $\Delta C_{9}$ is a volume effect, reflecting the fact that the posterior is slightly broader in $C_{7}$ and $C_{10}$ at values below the best-fit $\Delta C_{9}$ than above it. The results show $\mathrm{a}>3 \sigma$ preference for a negative offset to the muonic version of the $C_{9}$ Wilson coefficient compared to the SM, consistent with recent results from other groups [127129]. These are largely driven by the $B^{0} \rightarrow K^{*} \mu^{+} \mu^{-}$angular observables, with the corresponding component of the best-fit likelihood improved by $\Delta \ln \mathcal{L}=13.2$ with respect to the $\mathrm{SM}$, and $\Delta \ln \mathcal{L}=9.8$ compared to the CMSSM. We can also see that $C_{7}$ is strongly constrained by $b \rightarrow s \gamma$ decays, to within $+0.04 /-0.03$ of its SM value.

\subsection{FlavBit standalone example}

GAMBIT modules can also be called directly from other codes as libraries, without actually needing to use GAMBIT itself. To do this, the calling code must specify the physics 

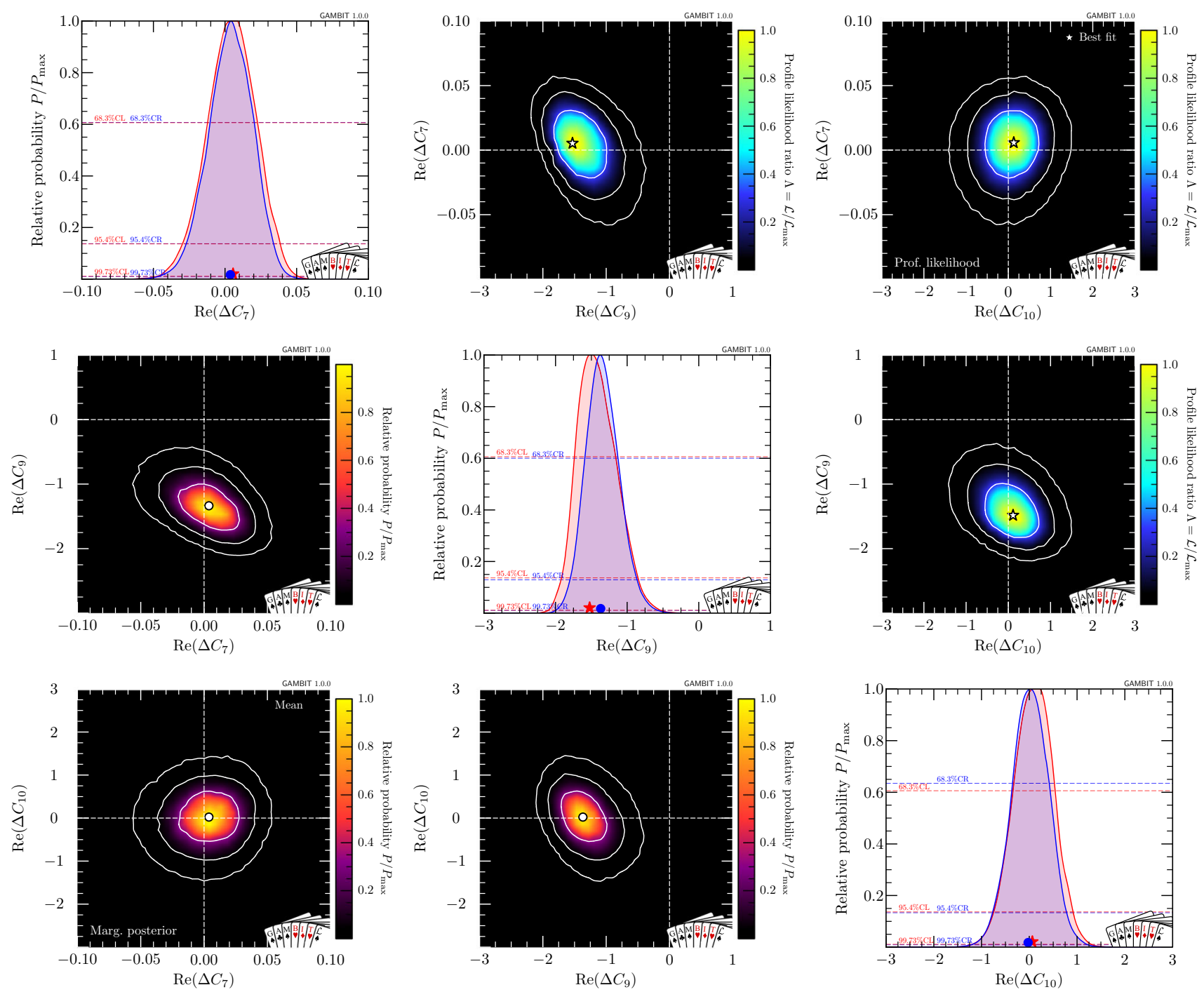

Fig. 2 Profile likelihoods (upper right panels) and posterior probabilities (bottom left panels) from a scan over the real parts of the Wilson coefficients $C_{7}, C_{9}$ and $C_{10}$, expressed in terms of the offsets $\Delta C_{i}$ from the SM values. The central diagonal shows both $1 \mathrm{D}$ posterior probabili-

model and parameter set to be used, the module and backend functions to be run, and any required options. The calling code is responsible for resolving the dependencies and backend requirements of each module function; this is typically done "by hand" by the author of the calling code, using simple GAMBIT utility functions to hardcode the links between the chosen module and backend functions. More details of using GAMBIT modules in this so-called 'standalone mode' can be found in Ref. [4].

An annotated driver program for calling FlavBit from outside the GAMBIT framework can be found in FlavBit/examples/FlavBit_standalone_example.cpp. As input, this program takes an SLHA file corresponding to the output of a spectrum generator (i.e. containing ties (blue) and profile likelihoods (red) for each parameter. Stars indicate the location of the best fit, filled circles indicate posterior means, and contours correspond to 1,2 , and $3 \sigma$ confidence. The SM prediction lies at the intersection of the dashed lines in the $2 \mathrm{D}$ panels

pole masses, $\overline{D R}$ parameters, etc). The name of this file can be given as a command-line argument. The program then calculates the full menu of FlavBit observables using Superlso 3.6 and FeynHiggs 2.11.3, and uses them to calculate the five independent FlavBit likelihoods. Much of this short program is dedicated to resolving module function dependencies and backend requirements. This includes defining a local function that creates a GAMBIT spectrum object from the input SLHA file, and others that fulfil the dependencies of SI_fill on the widths of the $Z$ and $W$ bosons.

If the user does not give the name of an input SLHA file when invoking the standalone example, it will read a default file given in the line 
sta: : string

infile("FlavBit/data/example.slha");

The likelihoods are retrieved in the lines

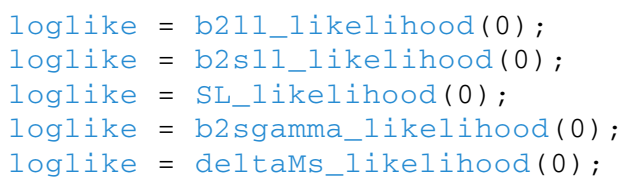

and can be combined or used for further analysis as the user requires.

The values of the observables, as used by the likelihoods, can be obtained directly from the respective observable functions in a similar manner, e.g.

double bsg = SI_bsgamma (0);

double Btaunu = SI_Btaunu $(0)$;

and so on for all observables in Tables 1, 2 and 3 .

\section{Conclusions}

In this paper we have described FlavBit, the flavour physics module of the public global-fitting framework GAMBIT. FlavBit provides calculations of a wide range of observables in flavour physics, ranging from tree-level decays of $B$ and $D$ mesons, to electroweak penguin decays, rare purely leptonic $B$ decays, $b \rightarrow s \gamma$ transitions, neutral meson oscillations, kaon and pion decays, and various isospin and forwardbackward asymmetries. These are so far implemented for supersymmetric and effective field theories, with the list of available theories expected to grow rapidly. FlavBit also features detailed experimental data, uncertainties, correlations and likelihood functions for tree-level leptonic and semileptonic, electroweak penguin, rare purely leptonic and $B \rightarrow X_{s} \gamma$ decays, as well as for the $B_{s}^{0}-\bar{B}_{s}^{0}$ mass difference.

We gave a number of interesting examples of FlavBit in action. These include a standalone example program that runs FlavBit without GAMBIT, in order to compute flavour observables in supersymmetry from an input SLHA file. We carried out an example supersymmetric flavour fit with FlavBit in GAMBIT, illustrating the impacts of its likelihoods. Finally, we performed a fit to a number of observables in the context of an effective theory of flavour, demonstrating about a $4 \sigma$ preference from combined experimental data for an approximately $25 \%$ deficit in the (muonic) $C_{9}$ Wilson coefficient, compared to the Standard Model prediction.

The FlavBit source code can be freely downloaded from gambit.hepforge.org, either as part of GAMBIT, or as a standalone package.

Acknowledgements We thank our colleagues within GAMBIT for many helpful discussions. We warmly thank the Casa Matemáti- cas Oaxaca, affiliated with the Banff International Research Station, for hospitality whilst part of this work was completed, and the staff at Cyfronet, for their always helpful supercomputing support. GAMBIT has been supported by STFC (UK; ST/K00414X/1, ST/P000762/1), the Royal Society (UK; UF110191), Glasgow University (UK; Leadership Fellowship), the Research Council of Norway (FRIPRO 230546/F20), NOTUR (Norway; NN9284K), the Knut and Alice Wallenberg Foundation (Sweden; Wallenberg Academy Fellowship), the Swedish Research Council (621-2014-5772), the Australian Research Council (CE110001004, FT130100018, FT140100244, FT160100274), The University of Sydney (Australia; IRCA-G162448), PLGrid Infrastructure (Poland), Polish National Science Center (Sonata UMO-2015/17/D/ST2/03532), the Swiss National Science Foundation (PP00P2-144674), the European Commission Horizon 2020 Marie Skłodowska-Curie actions (H2020-MSCA-RISE-2015-691164), the ERA-CAN+ Twinning Program (EU \& Canada), the Netherlands Organisation for Scientific Research (NWO-Vidi 680-47-532), the National Science Foundation (USA; DGE-1339067), the FRQNT (Québec) and NSERC/The Canadian Tri-Agencies Research Councils (BPDF-424460-2012).

Open Access This article is distributed under the terms of the Creative Commons Attribution 4.0 International License (http://creativecomm ons.org/licenses/by/4.0/), which permits unrestricted use, distribution, and reproduction in any medium, provided you give appropriate credit to the original author(s) and the source, provide a link to the Creative Commons license, and indicate if changes were made.

Funded by SCOAP ${ }^{3}$.

\section{Appendix A: Glossary}

Here we explain some terms that have specific technical definitions in GAMBIT.

backend An external code containing useful functions (or variables) that one might wish to call (or read/write) from a module function.

backend function $\mathrm{A}$ function contained in a backend. It calculates a specific quantity indicated by its capability. Its capability and call signature are defined in the backend's frontend header.

backend requirement $A$ declaration that a given module function needs to be able to call a backend function or use a backend variable, identified according to its capability and type(s). Backend requirements are declared in module functions' entries in rollcall headers.

backend variable $A$ global variable contained in a backend. It corresponds to a specific quantity indicated by its capability. Its capability and type are defined in the backend's frontend header.

capability A name describing the actual quantity that is calculated by a module or backend function. This is one possible place for units to be noted; the other is in the documented description of the capability (see Sec. 10.7 of Ref. [4]).

dependency A declaration that a given module function needs to be able to access the result of another module 
function, identified according to its capability and type. Dependencies are declared in module functions' entries in rollcall headers.

dependency resolution The process by which GAMBIT determines the module functions, backend functions and backend variables needed and allowed for a given scan, connects them to each others' dependencies and backend requirements, and determines the order in which they must be called.

frontend The interface between GAMBIT and a given backend, consisting of a frontend header plus optional source files and type headers.

frontend header The $\mathrm{C}++$ header in which the frontend to a given backend is declared.

module A subset of GAMBIT functions following a common theme, able to be compiled into a standalone library. Although module often gets used as shorthand for physics module, this term technically also includes the GAMBIT scanning module ScannerBit.

module function A function contained in a physics module. It calculates a specific quantity indicated by its capability and type, as declared in the module's rollcall header. It takes only one argument, by reference (the quantity to be calculated), and has a void return type.

physics module Any module other than ScannerBit, containing a collection of module functions following a common physics theme.

rollcall header The $\mathrm{C}++$ header in which a given physics module and its module functions are declared.

type A general fundamental or derived $\mathrm{C}++$ type, often referring to the type of the capability of a module function.

\section{References}

1. N. Serra, R. Silva, Coutinho, D. van Dyk, Measuring the breaking of lepton flavor universality in $B \rightarrow K^{*} \ell^{+} \ell^{-}$. Phys. Rev. D 95, 035029 (2017). arXiv: 1610.08761

2. D. Straub, flav-io/flavio v0.20.3 (2017). https://doi.org/10.5281/ zenodo. 438351

3. E.L. Silvestrini, HEPfit: a code for the combination of indirect and direct constraints on high energy physics models (2017). http:// hepfit.roma1.infn.it/

4. GAMBIT Collaboration: P. Athron, C. Balazs et al., GAMBIT: the global and modular beyond-the-standard-model inference tool. Eur. Phys. J. C (2017). arXiv: 1705.07908

5. F. Mahmoudi, SuperIso: a program for calculating the isospin asymmetry of $B \rightarrow K^{*} \gamma$ in the MSSM. Comput. Phys. Commun. 178, 745 (2008). arXiv:0710.2067

6. F. Mahmoudi, SuperIso v2.3: a program for calculating flavor physics observables in supersymmetry. Comput. Phys. Commun. 180, 1579 (2009). arXiv:0808.3144

7. F. Mahmoudi, SuperIso v3.0, flavor physics observables calculations: extension to NMSSM. Comput. Phys. Commun. 180, 1718 (2009)
8. GAMBIT Scanner Workgroup: G.D. Martinez, J. McKay et al., Comparison of statistical sampling methods with ScannerBit, the GAMBIT scanning module. Eur. Phys. J. C (2017). arXiv:1705.07959

9. GAMBIT Collaboration: P. Athron, C. Balázs et al., Status of the scalar singlet dark matter model. Eur. Phys. J. C 77(8), 568 (2017). arXiv: 1705.07931

10. GAMBIT Collaboration: P. Athron, C. Balázs et al., Global fits of GUT-scale SUSY models with GAMBIT. https://doi.org/10. 1140/epjc/s10052-017-5167-0. arXiv:1705.07935

11. GAMBIT Collaboration: P. Athron, C. Balázs et al., A global fit of the MSSM with GAMBIT. Eur. Phys. J. C (2017). arXiv: 1705.07917

12. GAMBIT Collider Workgroup: C. Balázs, A. Buckley et al., ColliderBit: a GAMBIT module for the calculation of high-energy collider observables and likelihoods. Eur. Phys. J. C (2017). arXiv: 1705.07919

13. GAMBIT Dark Matter Workgroup: T. Bringmann, J. Conrad et al., DarkBit: a GAMBIT module for computing dark matter observables and likelihoods. Eur. Phys. J. C (2017). arXiv:1705.07920

14. GAMBIT Models Workgroup: P. Athron, C. Balázs et al., SpecBit, DecayBit and PrecisionBit: GAMBIT modules for computing mass spectra, particle decay rates and precision observables. Eur. Phys. J. C (2017). arXiv:1705.07936

15. LHCb Collaboration: R. Aaij et al., Angular analysis and differential branching fraction of the decay $B_{s}^{0} \rightarrow \phi \mu^{+} \mu^{-}$. JHEP 09, 179 (2015). arXiv: 1506.08777

16. LHCb Collaboration: R. Aaij et al., Measurement of the ratio of branching fractions $\mathcal{B}\left(\bar{B}^{0} \rightarrow D^{*+} \tau^{-} \bar{v}_{\tau}\right) / \mathcal{B}\left(\bar{B}^{0} \rightarrow D^{*+} \mu^{-} \bar{v}_{\mu}\right)$. Phys. Rev. Lett. 115, 111803 (2015). arXiv:1506.08614 [Addendum: Phys. Rev. Lett. 115(15), 159901 (2015)]

17. LHCb Collaboration: R. Aaij et al., Test of lepton universality using $B^{+} \rightarrow K^{+} \ell^{+} \ell^{-}$decays. Phys. Rev. Lett. 113, 151601 (2014). arXiv:1406.6482

18. LHCb Collaboration: R. Aaij et al., Measurement of form-factorindependent observables in the decay $B^{0} \rightarrow K^{* 0} \mu^{+} \mu^{-}$. Phys. Rev. Lett. 111, 191801 (2013). arXiv:1308.1707

19. BaBar Collaboration: J.P. Lees et al., Evidence for an excess of $\bar{B} \rightarrow D^{(*)} \tau^{-} \bar{\nu}_{\tau}$ decays. Phys. Rev. Lett. 109, 101802 (2012). arXiv: 1205.5442

20. BaBar Collaboration: J.P. Lees et al., Measurement of an excess of $\bar{B} \rightarrow D^{(*)} \tau^{-} \bar{\nu}_{\tau}$ decays and implications for charged Higgs bosons. Phys. Rev. D 88, 072012 (2013). arXiv:1303.0571

21. Belle Collaboration: M. Huschle et al., Measurement of the branching ratio of $\bar{B} \rightarrow D^{(*)} \tau^{-} \bar{\nu}_{\tau}$ relative to $\bar{B} \rightarrow D^{(*)} \ell^{-} \bar{\nu}_{\ell}$ decays with hadronic tagging at Belle. Phys. Rev. D 92, 072014 (2015). arXiv:1507.03233

22. Belle Collaboration: A. Abdesselam et al., Measurement of the branching ratio of $\bar{B}^{0} \rightarrow D^{*+} \tau^{-} \bar{v}_{\tau}$ relative to $\bar{B}^{0} \rightarrow D^{*+} \ell^{-} \bar{v}_{\ell}$ decays with a semileptonic tagging method. In: Proceedings, 51st Rencontres de Moriond on Electroweak Interactions and Unified Theories, La Thuile, Italy (2016). arXiv:1603.06711

23. Belle Collaboration: A. Abdesselam et al., Angular analysis of $B^{0} \rightarrow K^{*}(892)^{0} \ell^{+} \ell^{-}$, in Proceedings, LHCSki 2016-A First Discussion of $13 \mathrm{TeV}$ Results: Obergurgl, Austria, April 10-15, 2016 (2016) arXiv: 1604.04042

24. M. Cacciari, G.P. Salam, G. Soyez, FastJet user manual. Eur. Phys. J. C 72, 1896 (2012). arXiv:1111.6097

25. P. Athron, J.-H. Park, D. Stöckinger, A. Voigt, FlexibleSUSY-a spectrum generator generator for supersymmetric models. Comput. Phys. Commun. 190, 139-172 (2015). arXiv:1406.2319

26. B.C. Allanach, SOFTSUSY: a program for calculating supersymmetric spectra. Comput. Phys. Commun. 143, 305-331 (2002). arXiv:hep-ph/0104145

27. S. Heinemeyer, W. Hollik, G. Weiglein, FeynHiggs: a program for the calculation of the masses of the neutral CP even Higgs bosons 
in the MSSM. Comput. Phys. Commun. 124, 76-89 (2000). arXiv:hep-ph/9812320

28. Particle Data Group: C. Patrignani et al., Review of particle physics. Chin. Phys. C 40, 100001 (2016)

29. Y. Sakaki, M. Tanaka, A. Tayduganov, R. Watanabe, Testing leptoquark models in $\bar{B} \rightarrow D^{(*)} \tau \bar{\nu}$. Phys. Rev. D 88, 094012 (2013). arXiv: 1309.0301

30. A. Crivellin, J. Heeck, P. Stoffer, A perturbed lepton-specific two-Higgs-doublet model facing experimental hints for physics beyond the Standard Model. Phys. Rev. Lett. 116, 081801 (2016). arXiv: 1507.07567

31. M. Freytsis, Z. Ligeti, J.T. Ruderman, Flavor models for $\bar{B} \rightarrow$ $D^{(*)} \tau \bar{\nu}$. Phys. Rev. D 92, 054018 (2015). arXiv:1506.08896

32. A.J. Buras, J. Girrbach, Towards the identification of new physics through quark flavour violating processes. Rep. Prog. Phys. 77, 086201 (2014). arXiv: 1306.3775

33. O. Eberhardt, U. Nierste, M. Wiebusch, Status of the two-Higgsdoublet model of type II. JHEP 07, 118 (2013). arXiv:1305.1649

34. D. Das, C. Hati, G. Kumar, N. Mahajan, Towards a unified explanation of $R_{D^{(*)}}, R_{K}$ and $(g-2)_{\mu}$ anomalies in a L-R model. Phys. Rev. D 94, 055034 (2016). arXiv:1605.06313

35. A. Greljo, G. Isidori, D. Marzocca, On the breaking of lepton flavor universality in B decays. JHEP 07, 142 (2015). arXiv: 1506.01705

36. S.M. Boucenna, A. Celis, J. Fuentes-Martin, A. Vicente, J. Virto, Phenomenology of an $S U(2) \times S U(2) \times U(1)$ model with lepton-flavour non-universality. JHEP 059, 1612 (2016). arXiv:1608.01349

37. D. Becirevic, N. Kosnik, O. Sumensari, R. Zukanovich Funchal, Palatable leptoquark scenarios for lepton flavor violation in exclusive $b \rightarrow s \ell_{1} \ell_{2}$ modes. JHEP 035, 1611 (2016). arXiv: 1608.07583

38. I. Dorsner, S. Fajfer, A. Greljo, J.F. Kamenik, N. Kosnik, Physics of leptoquarks in precision experiments and at particle colliders. Phys. Rep. 641, 1-68 (2016). arXiv:1603.04993

39. UTfit Collaboration: M. Bona et al., An improved standard model prediction of $\mathrm{BR}(B \rightarrow \tau \nu)$ and its implications for new physics. Phys. Lett. B 687, 61-69 (2010). arXiv:0908.3470

40. A.G. Akeroyd, F. Mahmoudi, Constraints on charged Higgs bosons from $D_{(s)}^{ \pm} \rightarrow \mu^{ \pm} v$ and $D_{(s)}^{ \pm} \rightarrow \tau^{ \pm} v$. JHEP 04, 121 (2009). arXiv:0902.2393

41. M. Beneke, T. Feldmann, D. Seidel, Systematic approach to exclusive $B \rightarrow V l^{+} l^{-}, V \gamma$ decays. Nucl. Phys. B 612, 25-58 (2001). arXiv:hep-ph/0106067

42. C. Greub, A. Ioannisian, D. Wyler, Effects of new physics in the rare decays $B \rightarrow K l^{+} l^{-}$and $B \rightarrow K^{*} l^{+} l^{-}$. Phys. Lett. B 346, 149-158 (1995). arXiv:hep-ph/9408382

43. F. Kruger, L.M. Sehgal, Lepton polarization in the decays $b \rightarrow$ $X_{(s)} \mu^{+} \mu^{-}$and $B \rightarrow X_{(s)} \tau^{+} \tau^{-}$. Phys. Lett. B 380, 199-204 (1996). arXiv:hep-ph/9603237

44. G. Hiller, F. Kruger, More model independent analysis of $b \rightarrow s$ processes. Phys. Rev. D 69, 074020 (2004). arXiv:hep-ph/0310219

45. LHCb Collaboration: R. Aaij et al., Differential branching fraction and angular analysis of the decay $B^{0} \rightarrow K^{* 0} \mu^{+} \mu^{-}$. JHEP 08, 131 (2013). arXiv: 1304.6325

46. J. Gratrex, M. Hopfer, R. Zwicky, Generalised helicity formalism, higher moments and the $B \rightarrow K_{J_{K}}(\rightarrow K \pi) \bar{\ell}_{1} \ell_{2}$ angular distributions. Phys. Rev. D 93, 054008 (2016). arXiv: 1506.03970

47. LHCb Collaboration: R. Aaij et al., Angular analysis of the $B^{0} \rightarrow$ $K^{* 0} \mu^{+} \mu^{-}$decay using $3 \mathrm{fb}^{-1}$ of integrated luminosity. JHEP $\mathbf{0 2}$, 104 (2016). arXiv: 1512.04442

48. F. Beaujean, M. ChrzÄĚszcz, N. Serra, D. van Dyk, Extracting angular observables without a likelihood and applications to rare decays. Phys. Rev. D 91, 114012 (2015). arXiv:1503.04100
49. W. Altmannshofer, P. Ball et al., Symmetries and asymmetries of $B \rightarrow K^{*} \mu^{+} \mu^{-}$decays in the standard model and beyond. JHEP 01, 019 (2009). arXiv:0811.1214

50. S. Descotes-Genon, T. Hurth, J. Matias, J. Virto, Optimizing the basis of $B \rightarrow K^{*} l l$ observables in the full kinematic range. JHEP 05, 137 (2013). arXiv:1303.5794

51. F. Kruger, J. Matias, Probing new physics via the transverse amplitudes of $B^{0} \rightarrow K^{* 0}\left(\rightarrow K^{-} \pi^{+}\right) l^{+} l^{-}$at large recoil. Phys. Rev. D 71, 094009 (2005). arXiv:hep-ph/0502060

52. D. Becirevic, E. Schneider, On transverse asymmetries in $B \rightarrow$ $K^{*} l^{+} l^{-}$. Nucl. Phys. B 854, 321-339 (2012). arXiv: 1106.3283

53. A. Bharucha, D.M. Straub, R. Zwicky, $B \rightarrow V \ell^{+} \ell^{-}$in the Standard Model from light-cone sum rules. JHEP 08, 098 (2016). arXiv: 1503.05534

54. S. JSger, J. Martin Camalich, On $B \rightarrow V \ell \ell$ at small dilepton invariant mass, power corrections, and new physics. JHEP $\mathbf{0 5}$, 043 (2013). arXiv: 1212.2263

55. J. Lyon, R. Zwicky, Resonances gone topsy turvy - the charm of QCD or new physics in $b \rightarrow s \ell^{+} \ell^{-}$? arXiv: 1406.0566

56. M. Ciuchini, M. Fedele et al., $B \rightarrow K^{*} \ell^{+} \ell^{-}$decays at large recoil in the Standard Model: a theoretical reappraisal. JHEP 06, 116 (2016). arXiv: 1512.07157

57. V.G. Chobanova, T. Hurth, F. Mahmoudi, D. Martinez Santos, S. Neshatpour, Large hadronic power corrections or new physics in the rare decay $B \rightarrow K^{*} \mu^{+} \mu^{-}$? JHEP 07, 025 (2017). arXiv: 1702.02234

58. S. Descotes-Genon, J. Matias, J. Virto, Understanding the $B \rightarrow K^{*} \mu^{+} \mu^{-}$anomaly. Phys. Rev. D 88, 074002 (2013). arXiv: 1307.5683

59. W. Altmannshofer, D.M. Straub, New physics in $B \rightarrow K^{*} \mu \mu$ ? Eur. Phys. J. C 73, 2646 (2013). arXiv: 1308.1501

60. T. Hurth, F. Mahmoudi, On the LHCb anomaly in $B \rightarrow K^{*} \ell^{+} \ell^{-}$. JHEP 04, 097 (2014). arXiv: 1312.5267

61. S. JSger, J. Martin Camalich, Reassessing the discovery potential of the $B \rightarrow K^{*} \ell^{+} \ell^{-}$decays in the large-recoil region: SM challenges and BSM opportunities. Phys. Rev. D 93, 014028 (2016). arXiv: 1412.3183

62. T. Hurth, F. Mahmoudi, S. Neshatpour, On the anomalies in the latest LHCb data. Nucl. Phys. B 909, 737-777 (2016). arXiv: 1603.00865

63. LHCb Collaboration: R. Aaij et al., Angular analysis of the $B^{0} \rightarrow K^{* 0} e^{+} e^{-}$decay in the low- ${ }^{2}$ region. JHEP 04, 064 (2015). arXiv:1501.03038

64. T. Feldmann, J. Matias, Forward-backward and isospin asymmetry for $B \rightarrow K^{*} \ell^{+} \ell^{-}$decay in the standard model and in supersymmetry. JHEP 1, 074 (2003). arXiv:hep-ph/0212158

65. T. Huber, T. Hurth, E. Lunghi, Inclusive $\bar{B} \rightarrow X_{s} \ell^{+} \ell^{-}$: complete angular analysis and a thorough study of collinear photons. JHEP 06, 176 (2015). arXiv:1503.04849

66. BaBar Collaboration: B. Aubert et al., Measurement of the $B \rightarrow$ $X_{s} \ell^{+} \ell^{-}$branching fraction with a sum over exclusive modes. Phys. Rev. Lett. 93, 081802 (2004). arXiv:hep-ex/0404006

67. Belle Collaboration: M. Iwasaki et al., Improved measurement of the electroweak penguin process $B \rightarrow X_{s} l^{+} l^{-}$. Phys. Rev. D 72, 092005 (2005). arXiv:hep-ex/0503044

68. Belle Collaboration: Y. Sato et al., Measurement of the lepton forward-backward asymmetry in $B \rightarrow X_{s} \ell^{+} \ell^{-}$decays with a sum of exclusive modes. Phys. Rev. D 93, 032008 (2016). arXiv:1402.7134 [Addendum: Phys. Rev. D 93(5), 059901 (2016)]

69. BaBar Collaboration: J.P. Lees et al., Measurement of the $B \rightarrow$ $X_{s} l^{+} l^{-}$branching fraction and search for direct CP violation from a sum of exclusive final states. Phys. Rev. Lett. 112, 211802 (2014). arXiv:1312.5364

70. LHCb Collaboration: R. Aaij et al., Differential branching fraction and angular moments analysis of the decay $B^{0} \rightarrow$ 
$K^{+} \pi^{-} \mu^{+} \mu^{-}$in the $K_{0,2}^{*}(1430)^{0}$ region. JHEP 12, 065 (2016). arXiv: 1609.04736

71. D. Das, G. Hiller, M. Jung, A. Shires, The $\bar{B} \rightarrow \bar{K} \pi \ell \ell$ and $\bar{B}_{s} \rightarrow \bar{K} K \ell \ell$ distributions at low hadronic recoil. JHEP 09, 109 (2014). arXiv:1406.6681

72. F. Mahmoudi, S. Neshatpour, J. Virto, $B \rightarrow K^{*} \mu^{+} \mu^{-}$optimised observables in the MSSM. Eur. Phys. J. C 74, 2927 (2014). arXiv: 1401.2145

73. S. Bertolini, F. Borzumati, A. Masiero, G. Ridolfi, Effects of supergravity induced electroweak breaking on rare $B$ decays and mixings. Nucl. Phys. B 353, 591-649 (1991)

74. M. Misiak et al., Estimate of $\mathcal{B}\left(\bar{B} \rightarrow X_{s} \gamma\right)$ at $O\left(\alpha_{s}^{2}\right)$. Phys. Rev. Lett. 98, 022002 (2007). arXiv:hep-ph/0609232

75. F. Mahmoudi, O. Stål, Flavor constraints on the two-Higgsdoublet model with general Yukawa couplings. Phys. Rev. D 81, 035016 (2010). arXiv:0907.1791

76. T. Hermann, M. Misiak, M. Steinhauser, $\bar{B} \rightarrow X_{s} \gamma$ in the two Higgs doublet model up to next-to-next-to-leading order in QCD. JHEP 11, 036 (2012). arXiv:1208.2788

77. M. Misiak et al., Updated NNLO QCD predictions for the weak radiative $B$-meson decays. Phys. Rev. Lett. 114, 221801 (2015). arXiv: 1503.01789

78. M. Misiak, M. Steinhauser, Weak radiative decays of the B meson and bounds on $M_{H^{ \pm}}$in the two-Higgs-doublet model. Eur. Phys. J. C 77, 201 (2017). arXiv:1702.04571

79. A.L. Kagan, M. Neubert, QCD anatomy of B $\rightarrow$ X(s gamma) decays. Eur. Phys. J. C 7, 5-27 (1999). arXiv:hep-ph/9805303

80. A.L. Kagan, M. Neubert, Isospin breaking in $B \rightarrow K^{*} \gamma$ decays. Phys. Lett. B 539, 227-234 (2002). arXiv:hep-ph/0110078

81. M.R. Ahmady, F. Mahmoudi, Constraints on the mSUGRA parameter space from NLO calculation of isospin asymmetry in $B \rightarrow K^{*} \gamma$. Phys. Rev. D 75, 015007 (2007). arXiv:hep-ph/0608212

82. M. González-Alonso, J. Martin Camalich, Global effective-fieldtheory analysis of new-physics effects in (semi)leptonic kaon decays. JHEP 12, 052 (2016). arXiv: 1605.07114

83. FlaviaNet Working Group on Kaon Decays: M. Antonelli et al., Precision tests of the Standard Model with leptonic and semileptonic kaon decays, in PHIPSI08, Proceedings of the International Workshop on $e^{+} e^{-}$Collisions from phi to psi, Frascati (Rome) Italy, 7-10 April 2008 (2008). arXiv:0801.1817

84. A.J. Buras, P.H. Chankowski, J. Rosiek, L. Slawianowska, $\Delta M_{d, s}, B^{0} d, s \rightarrow \mu^{+} \mu^{-}$and $B \rightarrow X_{s} \gamma$ in supersymmetry at large $\tan \beta$. Nucl. Phys. B 659, 3 (2003). arXiv:hep-ph/0210145

85. BaBar Collaboration: B. Aubert et al., A measurement of the branching fractions of exclusive $\bar{B} \rightarrow D^{(*)}(\pi) \ell^{-} \bar{v}(\ell)$ decays in events with a fully reconstructed $B$ meson. Phys. Rev. Lett. 100, 151802 (2008). arXiv:0712.3503

86. BaBar Collaboration: B. Aubert et al., Measurement of $|V(c b)|$ and the form-factor slope in anti-B $\rightarrow$ D l-anti-nu decays in events tagged by a fully reconstructed B meson. Phys. Rev. Lett. 104, 011802 (2010). arXiv:0904.4063

87. Belle Collaboration: W. Dungel et al., Measurement of the form factors of the decay $B^{0} \rightarrow D^{*-} \ell^{+} v$ and determination of the CKM matrix element $|V c b|$. Phys. Rev. D 82, 112007 (2010). arXiv: 1010.5620

88. Belle Collaboration: R. Glattauer et al., Measurement of the decay $B \rightarrow D \ell \nu_{\ell}$ in fully reconstructed events and determination of the Cabibbo-Kobayashi-Maskawa matrix element $\left|V_{c b}\right|$. Phys. Rev. D 93, 032006 (2016). arXiv: 1510.03657

89. Belle Collaboration: Y. Sato et al., Measurement of the branching ratio of $\bar{B}^{0} \rightarrow D^{*+} \tau^{-} \bar{v}_{\tau}$ relative to $\bar{B}^{0} \rightarrow D^{*+} \ell^{-} \bar{v}_{\ell}$ decays with a semileptonic tagging method. Phys. Rev. D 94, 072007 (2016). arXiv: 1607.07923
90. Belle Collaboration: S. Hirose et al., Measurement of the $\tau$ lepton polarization and $R\left(D^{*}\right)$ in the decay $\bar{B} \rightarrow D^{*} \tau^{-} \bar{v}_{\tau}$. Phys. Rev. Lett. 118, 211801 (2017). arXiv: 1612.00529

91. Y. Amhis et al., Averages of $b$-hadron, $c$-hadron, and $\tau$-lepton properties as of summer 2016. arXiv: 1612.07233

92. Y. Amhis et al., Average of $R(D)$ and $R\left(D^{*}\right)$ for Moriond EW 2017. http://www.slac.stanford.edu/xorg/hfag/semi/moriond17/ RDRDs.html

93. HPQCD Collaboration: H. Na, C.M. Bouchard, G.P. Lepage, C. Monahan, J. Shigemitsu, $B \rightarrow D l v$ form factors at nonzero recoil and extraction of $\left|V_{c b}\right|$. Phys. Rev. D 92, 054510 (2015). arXiv:1505.03925. [Erratum: Phys. Rev. D 93(11), 119906 (2016)]

94. S. Fajfer, J.F. Kamenik, I. Nisandzic, On the $B \rightarrow D^{*} \tau \bar{\nu}_{\tau}$ sensitivity to new physics. Phys. Rev. D 85, 094025 (2012). arXiv: 1203.2654

95. S. Fajfer, J.F. Kamenik, I. Nišandžić, $b \rightarrow D^{*} \tau \bar{v}_{\tau}$. Phys. Rev. D 85, 094025 (2012)

96. Fermilab Lattice, MILC: J.A. Bailey et al., $\left|V_{u b}\right|$ from $B \rightarrow \pi \ell \nu$ decays and (2+1)-flavor lattice QCD. Phys. Rev. D 92, 014024 (2015). arXiv: 1503.07839

97. BaBar Collaboration: J.P. Lees et al., Evidence of $B^{+} \rightarrow \tau^{+} v$ decays with hadronic B tags. Phys. Rev. D 88, 031102 (2013). arXiv: 1207.0698

98. BaBar Collaboration: B. Aubert et al., A search for $B^{+} \rightarrow \ell^{+} v_{\ell}$ recoiling against $B^{-} \rightarrow D^{0} \ell^{-} \bar{v} X$. Phys. Rev. D 81, 051101 (2010). arXiv:0912.2453

99. Belle Collaboration: I. Adachi et al., Evidence for $B^{-} \rightarrow \tau^{-} \bar{v}_{\tau}$ with a hadronic tagging method using the full data sample of Belle. Phys. Rev. Lett. 110, 131801 (2013). arXiv:1208.4678

100. Belle Collaboration: K. Hara et al., Evidence for $B^{-} \rightarrow \tau^{-} \bar{v}$ with a semileptonic tagging method. Phys. Rev. D 82, 071101 (2010). arXiv: 1006.4201

101. ATLAS Collaboration, Angular analysis of $B_{d}^{0} \rightarrow K^{*} \mu^{+} \mu^{-}$ decays in $p p$ collisions at $\sqrt{s}=8 \mathrm{TeV}$ with the ATLAS detector, in 52nd Rencontres de Moriond on Electroweak Interactions and Unified Theories (2017). ATLAS-CONF-2017-023

102. CMS Collaboration, Measurement of the $P_{1}$ and $P_{5}^{\prime}$ angular parameters of the decay $\mathrm{B}^{0} \rightarrow \mathrm{K}^{* 0} \mu^{+} \mu^{-}$in proton-proton collisions at $\sqrt{s}=8 \mathrm{TeV}$, in 52nd Rencontres de Moriond on Electroweak Interactions and Unified Theories (2017). CMS-PASBPH-15-008

103. F. Mahmoudi, T. Hurth, S. Neshatpour, Present status of $b \rightarrow$ $s l^{+} l^{-}$anomalies, in 6th Workshop on Theory, Phenomenology and Experiments in Flavour Physics: Interplay of Flavour Physics with electroweak symmetry breaking (Capri 2016) Anacapri, Capri, Italy, June 11, 2016 (2016). arXiv:1611.05060

104. LHCb Collaboration: R. Aaij et al., Measurement of the $B_{s}^{0} \rightarrow$ $\mu^{+} \mu^{-}$branching fraction and effective lifetime and search for $B^{0} \rightarrow \mu^{+} \mu^{-}$decays. Phys. Rev. Lett. 118, 191801 (2017). arXiv: 1703.05747

105. LHCb and CMS Collaborations: V. Khachatryan et al., Observation of the rare $B_{s}^{0} \rightarrow \mu^{+} \mu^{-}$decay from the combined analysis of CMS and LHCb data. Nature 522, 68-72 (2015). arXiv: 1411.4413

106. LHCb Collaboration: R. Aaij et al., Measurement of the $B_{s}^{0} \rightarrow$ $\mu^{+} \mu^{-}$branching fraction and search for $B^{0} \rightarrow \mu^{+} \mu^{-}$decays at the LHCb experiment. Phys. Rev. Lett. 111, 101805 (2013). arXiv: 1307.5024

107. CMS Collaboration: S. Chatrchyan et al., Measurement of the $B_{s}^{0} \rightarrow \mu^{+} \mu^{-}$branching fraction and search for $B^{0} \rightarrow \mu^{+} \mu^{-}$ with the CMS experiment. Phys. Rev. Lett. 111, 101804 (2013). arXiv: 1307.5025

108. ATLAS Collaboration: M. Aaboud et al., Study of the rare decays of $B_{s}^{0}$ and $B^{0}$ into muon pairs from data collected during the LHC 
Run 1 with the ATLAS detector. Eur. Phys. J. C 76(9), 513 (2016). arXiv: 1604.04263

109. CDF Collaboration: T. Aaltonen et al., Search for the decays $B_{s}^{0} \rightarrow e^{+} \mu^{-}$and $B_{s}^{0} \rightarrow e^{+} e^{-}$in CDF Run II. Phys. Rev. Lett. 102, 201801 (2009). arXiv:0901.3803

110. LHCb Collaboration: R. Aaij et al., Search for the decays $B_{s}^{0} \rightarrow$ $\tau^{+} \tau^{-}$and $B^{0} \rightarrow \tau^{+} \tau^{-}$. Phys. Rev. Lett. 118, 251802 (2017) arXiv: 1703.02508

111. BaBar Collaboration: B. Aubert et al., A search for the rare decay $B^{0} \rightarrow \tau^{+} \tau^{-}$at BABAR. Phys. Rev. Lett. 96, 241802 (2006). arXiv:hep-ex/0511015

112. A.J. Buras, J. Girrbach, D. Guadagnoli, G. Isidori, On the Standard Model prediction for $\operatorname{BR}\left(B_{s, d} \rightarrow \mu^{+} \mu^{-}\right)$. Eur. Phys. J. C 72, 2172 (2012). arXiv:1208.0934

113. BaBar Collaboration: B. Aubert et al., Measurement of the $B \rightarrow$ $X_{s} \gamma$ branching fraction and photon energy spectrum using the recoil method. Phys. Rev. D 77, 051103 (2008). arXiv:0711.4889

114. BaBar Collaboration: J.P. Lees et al., Exclusive measurements of $b \rightarrow s \gamma$ transition rate and photon energy spectrum. Phys. Rev. D 86, 052012 (2012). arXiv: 1207.2520

115. BaBar Collaboration: J.P. Lees et al., Precision measurement of the $B \rightarrow X_{s} \gamma$ photon energy spectrum, branching fraction, and direct CP asymmetry $A_{C P}\left(B \rightarrow X_{s+d} \gamma\right)$. Phys. Rev. Lett. 109, 191801 (2012). arXiv: 1207.2690

116. Belle Collaboration: T. Saito et al., Measurement of the $\bar{B} \rightarrow X_{s} \gamma$ branching fraction with a sum of exclusive decays. Phys. Rev. D 91, 052004 (2015). arXiv:1411.7198

117. Belle Collaboration: A. Abdesselam et al., Measurement of the inclusive $B \rightarrow X_{s+d} \gamma$ branching fraction, photon energy spectrum and HQE parameters, in Proceedings, 38th International Conference on High Energy Physics (ICHEP 2016): Chicago, IL, USA, August 3-10, 2016 (2016). arXiv:1608.02344

118. M. Czakon, P. Fiedler et al., The $\left(Q_{7}, Q_{1,2}\right)$ contribution to $\bar{B} \rightarrow$ $X_{s} \gamma$ at $\mathcal{O}\left(\alpha_{\mathrm{s}}^{2}\right)$. JHEP 04, 168 (2015). arXiv: 1503.01791
119. M. Artuso, G. Borissov, A. Lenz, CP violation in the $B_{s}^{0}$ system. Rev. Mod. Phys. 88, 045002 (2016). arXiv:1511.09466

120. KLOE Collaboration: F. Ambrosino et al., Precise measurement of $\Gamma(K \rightarrow e v(\gamma)) / \Gamma(K \rightarrow \mu \nu(\gamma))$ and study of $K \rightarrow e v \gamma$. Eur. Phys. J. C 64, 627-636 (2009). arXiv:0907.3594 [Erratum: Eur. Phys. J. C 65, 703 (2010)]

121. NA62 Collaboration: C. Lazzeroni et al., Precision measurement of the ratio of the charged kaon leptonic decay rates. Phys. Lett. B 719, 326-336 (2013). arXiv: 1212.4012

122. Y. Akrami, P. Scott, J. Edsjö, J. Conrad, L. Bergström, A profile likelihood analysis of the constrained MSSM with genetic algorithms. JHEP 4, 57 (2010). arXiv:0910.3950

123. F. Feroz, K. Cranmer, M. Hobson, R. Ruiz de Austri, R. Trotta, Challenges of profile likelihood evaluation in multi-dimensional SUSY scans. JHEP 6, 42 (2011). arXiv: 1101.3296

124. A. Arbey, M. Battaglia, F. Mahmoudi, D. Martinez Santos, Supersymmetry confronts $B_{S} \rightarrow \mu^{+} \mu^{-}$: present and future status. Phys. Rev. D 87, 035026 (2013). arXiv: 1212.4887

125. J. Skilling, Nested sampling, in American Institute of Physics Conference Series, ed. by R. Fischer, R. Preuss, U.V. Toussaint, vol 735 (2004), pp. 395-405

126. F. Feroz, M.P. Hobson, M. Bridges, MULTINEST: an efficient and robust Bayesian inference tool for cosmology and particle physics. MNRAS 398, 1601-1614 (2009). arXiv:0809.3437

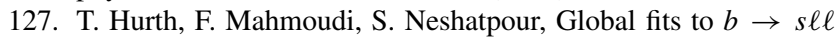
data and signs for lepton non-universality. JHEP 12, 053 (2014). arXiv: 1410.4545

128. W. Altmannshofer, C. Niehoff, P. Stangl, D.M. Straub, Status of the $B \rightarrow K^{*} \mu^{+} \mu^{-}$anomaly after Moriond. Eur. Phys. J. C 77(6), 377 (2017). arXiv:1703.09189

129. S. Descotes-Genon, L. Hofer, J. Matias, J. Virto, Global analysis of $b \rightarrow$ s $\ell \ell$ anomalies. JHEP 06, 092 (2016). arXiv:1510.04239 